\title{
Food and Nutrient Intakes Assessed with Dietary Records for the Validation Study of a Self-administered Food Frequency Questionnaire in JPHC Study Cohort I
}

\author{
Satoshi Sasaki, ${ }^{1}$ Tosei Takahashi, ${ }^{2}$ Yoji litoi, ${ }^{2}$ Yasuhiko Iwase, ${ }^{3}$ Minatsu Kobayashi, ${ }^{1}$ Junko Ishihara, ${ }^{4}$ \\ Masayuki Akabane, ${ }^{2}$ and Shoichiro Tsugane ${ }^{1}$
}

\begin{abstract}
We present here the survey methods and basic results of dietary records which were used as reference values in the present validation study of a self-administered food frequency questionnaire (FFQ) for the 5-year follow-up survey of the JPHC study. A semi-weighed dietary record was kept for four seven consecutive days in each of the four seasons in 3 areas, i.e., Iwate, Akita, and Nagano, and for seven consecutive days in both winter and summer in Okinawa. The mean intakes were significantly different between areas for some nutrients and food groups. A significant seasonal difference in the mean intakes was also observed in some nutrients such as carotene and vitamin $\mathrm{C}$, and in some food groups such as potatoes, vegetables, and fruits in both sexes, and alcoholic beverages in men and milks in women $(p<0.001)$. J Epidemiol 2003; 13(suppl):S23-S50.
\end{abstract}

Key words: weighed dietary record, nutrient, food group, diet.

Food and nutrient intakes have frequently been used as the "gold standard" in several validation studies of newly developed dietary assessment questionnaires. In this validation study of a self-administered semiquantitative food frequency questionnaire (FFQ) used in the 5-year follow-up survey of the JPHC study, 28day dietary records (14-day records in Okinawa) were collected from the sample populations living in 4 cohort areas (Iwate, Akita, Nagano, and Okinawa). In this paper, brief computation methods and the area-based intake levels of main food groups and nutrients are presented as basic data for the subsequent results of the validation study. The basic results on seasonal variation were also presented for nutrients and food groups.

\section{METHODS}

\section{Data Collection}

Semi-weighed dietary records of four different seasons over seven consecutive days were collected by a method used in the National Nutrition Survey (NNS)' with some modifications. Research dietitians instructed the subjects to record all foods and beverages prepared and consumed in a specially designed booklet. The participants were asked to provide detailed descriptions of each food, including the methods of preparation and recipes whenever possible. The dieticians checked the records at each participant's home during the survey and reviewed them in a standardized way after recording.

\section{Coding}

All foods ingested were coded with food codes by trained dietitians mainly in one laboratory. Some cooked foods and menus for which it was difficult to estimate the food items used in the preparation were coded with prepared cooked foods/menu codes by the authors and converted into food items after the coding using the standard recipe database developed by the authors. For rice, wellmilled rice was used for the computation. Water including cooking water such as water added for rice-boiling and for miso (fermented soybean paste)-soup was not included in the coding. The weight in grams was also coded for all foods ingested except for cooking water and some beverages. Dietary supplements were coded using temporary codes prepared by the authors, but the weights were not coded.

\section{Computation of Food and Nutrient Intakes}

Nutrient intakes were calculated using the Standard Tables of Food Composition in Japan, the 4 th revised edition ${ }^{2}$ for energy and 16 nutrients. As the food composition table for cholesterol

\footnotetext{
1 Epidemiology and Biostatistics Division, National Cancer Center Research institute East.

2 Department of Nutrition, Tokyo University of Agriculture.

3 Department of Applied Bioscience. Tokyo University of Agriculture.

- Cancer information and Epidemiology Division, National Cancer Center Research Institute.

Address for correspondence : Shoichiro Tsugane, Epidemiology and Biostatistics Division, National Cancer Center Research Institute East, 6-5-1 Kashiwanoha, Kashiwa, Chiba 277-8577, Japan.
} 
has numerous missing values, ${ }^{3}$ one was developed applying the substituting methods used for the development of fatty acid food composition table. ${ }^{4}$ Cooking oils were coded as "vegetable oil" without specific type and/or brand name. For the calculation of fatty acid intakes, "vegetable oil, mixed" (mixture of $30 \%$ of rapeseed oil and $70 \%$ of soybean oil) was used for all the cooking oils appearing in the data. Rice was converted into boiled rice multiplied by 2.25 for comparison with the results obtained from FFQ.

Green and yellow vegetables were defined as 44 vegetables with 600 micrograms of carotene per 100 gram and 10 frequently used vegetables that contribute to the intake of carotene among Japanese, according to the definition by the Ministry of Health, Labor and Welfare. Additionally, mugwort (leaves) with 3600 micro grams of carotene per 100 gram food portion was included in green and yellow vegetables because it was not negligible as a carotene source among the subjects in Okinawa in a previous survey ${ }^{5}$. Pickled vegetables were also computed separately from total vegetables. Pickled plum was included in the pickled vegetables, whereas it was otherwise categorized as a fruit.

Among non-alcoholic beverages, only green tea brewed at home was reported without the number of cups drunken. The amount consumed was taken to be $200 \mathrm{~g}$ each time.

\section{Statistical Analysis}

The 215 subjects (102 men and 113 women), both with FFQ for the validation study and the complete DR (14-day records in Okinawa and 28-day records in the other 3 areas), were included in this analysis. Mean intakes by PHC area were presented, and the difference among the 4 areas was examined using analysis of variance (ANOVA). In order to examine a possible seasonal variation of food and nutrient intakes, the mean intakes by season are also presented, and the seasonal difference was examined using two-way ANOVA adjusting for area difference. In this analysis, the data from Okinawa were excluded because of the lack of data for the survey in spring and autumn. The comparison of mean intakes between winter and summer was performed including all 4 areas using paired t-test. Intakes of nutrients and foods were adjusted for energy intake using the energy density method to eliminate possible association between intakes and total caloric intakes. The top 20 foods for each nutrient were listed, and their contributions to the nutrient were calculated.

\section{Data for Comparison}

In order to examine different intake levels from general Japanese populations, we compared them with the published data of the National Nutrition Survey (NNS) in 1995'. The mean intakes of food group and selected nutrients were reported by agerange (every 10 years) and sex, and the mean intakes age-adjusted for the study subjects by sex were used in this study. Because of a slightly different definition of food groups and survey methods, some food groups such as pickled vegetables, beverages, and seasonings and spices were not compared in the two surveys. Mean total intakes of nutrient and food groups were compared with the results from the NNS by dividing the difference between mean intake of our study and the NNS by mean intake of the NNS (percent difference).

\section{RESULTS}

Tables 1 and 2 show the intake levels of energy and main nutrients by sex and area. The mean energy intake was highest in the Ninohe PHC area, and lowest in the Ishikawa PHC area for both men and women. Mean intakes were significantly different among areas for most of the nutrients except alcohol, retinol, niacin and vitamin $C$ for both sexes, and for total fat, carotene, vitamin $B 1$ and $B_{2}$ for women ( $p<0.05$ ). Mean intakes were close to the values reported in the NNS among similar age-groups in the same year,' i.e., difference within $10 \%$ for all nutrients examined except for sodium and vitamin $\mathrm{C}$ in both sexes. After adjustment for energy, the area differences were significant only for total fat, carbohydrate, calcium, sodium, carotene, niacin for both sexes, and for vitamin $B_{2}$ and $C$ for men $(p<0.05)$.

Tables 3 and 4 show the intake levels of main nutrients by season and sex. A significant seasonal difference was observed in the mean intake of iron, carotene and vitamin $\mathrm{C}$ in both men and women, and in the mean intake of carbohydrate, calcium, sodium, and retinol in women $(\mathrm{p}<0.05)$ when the crude intakes in the 3 areas were examined. When the values in the 4 areas were examined between summer and winter, a significant seasonal difference was observed in the mean intake of iron and carotene in both sexes, and for calcium, retinol and vitamin $B_{1}$ in women. When the energy-adjustment values in the 3 areas were examined, a significant seasonal difference was observed in the mean intake of protein, iron, potassium, carotene, niacin and vitamin $\mathrm{C}$ in men, and in total fat, carbohydrate, calcium, iron, sodium, potassium, retinol, carotene, vitamin $\mathrm{B} 2$, vitamin $\mathrm{C}$ in women $(\mathbf{p}<0.05)$. When the energy-adjusted values in 4 areas were examined between summer and winter, a significant seasonal difference was observed in the mean intake of protein, iron, potassium and retinol in men, and in protein, calcium, iron, potassium, retinol, carotene, vitamin $B_{1}$ and vitamin $B_{2}$ in women.

Tables 5 and 6 show the intake levels of food groups by sex and area. Mean intakes were significantly different among the 4 areas in most of the food groups except for potatoes and starches, and alcoholic and nonalcoholic beverages in both sexes, algae in men, and green and yellow vegetables, and milks in women $(p<0.05)$. The difference between the observed intakes and the reported ones from the NNS was $040 \%$ to $60 \%$ larger than that observed for nutrients. After the adjustment for energy, the area differences were significant for most of the food groups with the exception of cereals, sugar and sweeteners, eggs, vegetables, algae and non-alcoholic beverages in men, and except for cereals, potatoes and starches, eggs, milks, vegetables, green and yellow vegetables, and alcoholic beverages in women $(\mathrm{p}<0.05)$.

Tables 7 and 8 show the intake levels of food groups by season and sex. A significant seasonal difference was observed in the 
mean intake of potatoes and starches, fats and oils, vegetables, green and yellow vegetables, pickled vegetables, fruits, fungi, algae, alcoholic beverages in men, and in the mean intake of potatoes and starches, fats and oils, pulses, milks, vegetables, green and yellow vegetables, pickled vegetables, fruits and fungi among women $(\mathrm{p}<0.05)$, when the crude values in the 3 areas were examined. In the 4 areas, a significant seasonal difference was observed between summer and winter in the mean intake of potatoes and starches, sugar and sweeteners, nuts and seeds, milks, vegetables, pickled vegetables, fruits, fungi, algae, and alcoholic beverages in men, and in the mean intake of potatoes and starches, fats and oils, nuts and seeds, pulses, milks, vegetables, green and yellow vegetables, pickled vegetables, fruits and fungi in women $(\mathrm{p}<0.05)$. This seasonal difference in 3 areas was consistent after energy adjustment for most food groups except confectioneries in men $(\mathrm{p}<0.05)$. The seasonal difference in 4 areas was also consistent for most food groups aside from milks and algae in men, and alcoholic beverages in women $(\mathrm{p}<0.05)$.

Tables 9-25 showed the cumulative percent contributions of the top 20 foods for each nutrient.

\section{DISCUSSION}

A wide variation was observed among the 4 areas both for food and nutrient intake levels at the population level. However, mean intakes of energy and the selected 7 nutrients were not markedly different from the mean values observed in the NNS during a similar period: the difference was within $10 \%$ for most of the nutrients compared. The area difference was less marked when energy-density values were used than when the crude values were used in the analysis.

Reports on seasonal differences in nutrient and food group intakes have been few in Japanese populations. ${ }^{6}$ Our data revealed a significant seasonal difference for more than a few nutrients and food groups in both crude and energy-density values. These findings were in general agreement with the previous reports in different areas of Japan. ${ }^{6}$ The data indicated the importance of considering seasonal differences in food group intakes and some specific nutrients such as carotene and vitamin $\mathrm{C}$, where long-term dietary habits are concerned. DRs from the Okinawa (Ishikawa PHC) area were collected only in winter and summer because the seasonal variation was expected to be minimal in a sub-tropical climate. Thus, seasonal differences in dietary habits in the Ishikawa PHC area were not investigated in the present study. When the intakes were compared between winter and summer including the data of the Ishikawa PHC area, the results were virtually consistent.

The top 20 foods contributing to intake of each nutrient in DRs were included in the FFQ food items, except for some which contributed to alcohol, carbohydrate, and sodium intakes. The lowest cumulative percentage among the top 20 foods was observed in potassium in men (46\%) and in women (44\%). The cumulative percentage among the top 20 foods was slightly lower in women than in men in most of the nutrients examined. The results were generally similar to those previously reported for a different population in Japan. ${ }^{7.8}$

\section{REFERENCES}

1. Ministry of Health and Welfare. Kokumin Eiyo no Genjou (Results of National Nutrition Survey, 1995). Tokyo: Daiichi Shuppan Publishers; 1997. (in Japanese)

2. Science and Technology Agency. Standard Tables of Food Composition in Japan. The fourth revised edition. Tokyo: Printing Bureau, Ministry of Finance; 1982. (in Japanese)

3. Science and Technology Agency. Fatty acids, cholesterol, vitamin E composition table of Japanese foods. Tokyo: Ishiyaku Shuppan Publishers; 1990. (in Japanese)

4. Sasaki S, Kobayashi M, Tsugane S. Development of substituted fatty acid food composition table for the use in nutritional epidemiologic studies for Japanese populations: its methodological background and the evaluation. J Epidemiol 1999;9:190-207.

5. Tsubono Y, Takamori S, Kobayashi M, Takahashi T, Iwase $\mathrm{Y}$, Iitoi $\mathrm{Y}$, et al. A data-based approach for designing a semiquantitative food frequency questionnaire for a populationbased prospective study in Japan. J Epidemiol 1995;6:45-53.

6. Owaki A, Takatsuka N, Kawakami N, Shimizu H. Seasonal variations of nutrient intake assessed by 24 -hour recall method. Jpn J Nutr 1996;54:11-8. (in Japanese with English abstract)

7. Tokudome Y, Imaeda N, Ikeda M, Kitagawa I, Fujiwara N, Tokudome S. Foods contributing to absolute intake and variance in intake of fat, fatty acids and cholesterol in middleaged Japanese. J Epidemiol 1999;9:780-90.

8. Imaeda N, Tokudome Y, Ikeda M, Kitagawa I, Fujiwara N, Tokudome S. Foods contributing to absolute intake and variance in intake of selected vitamins, minerals and dietary fiber in middle-aged Japanese. J Nutr Sci Vitaminol 1999;45:51932. 


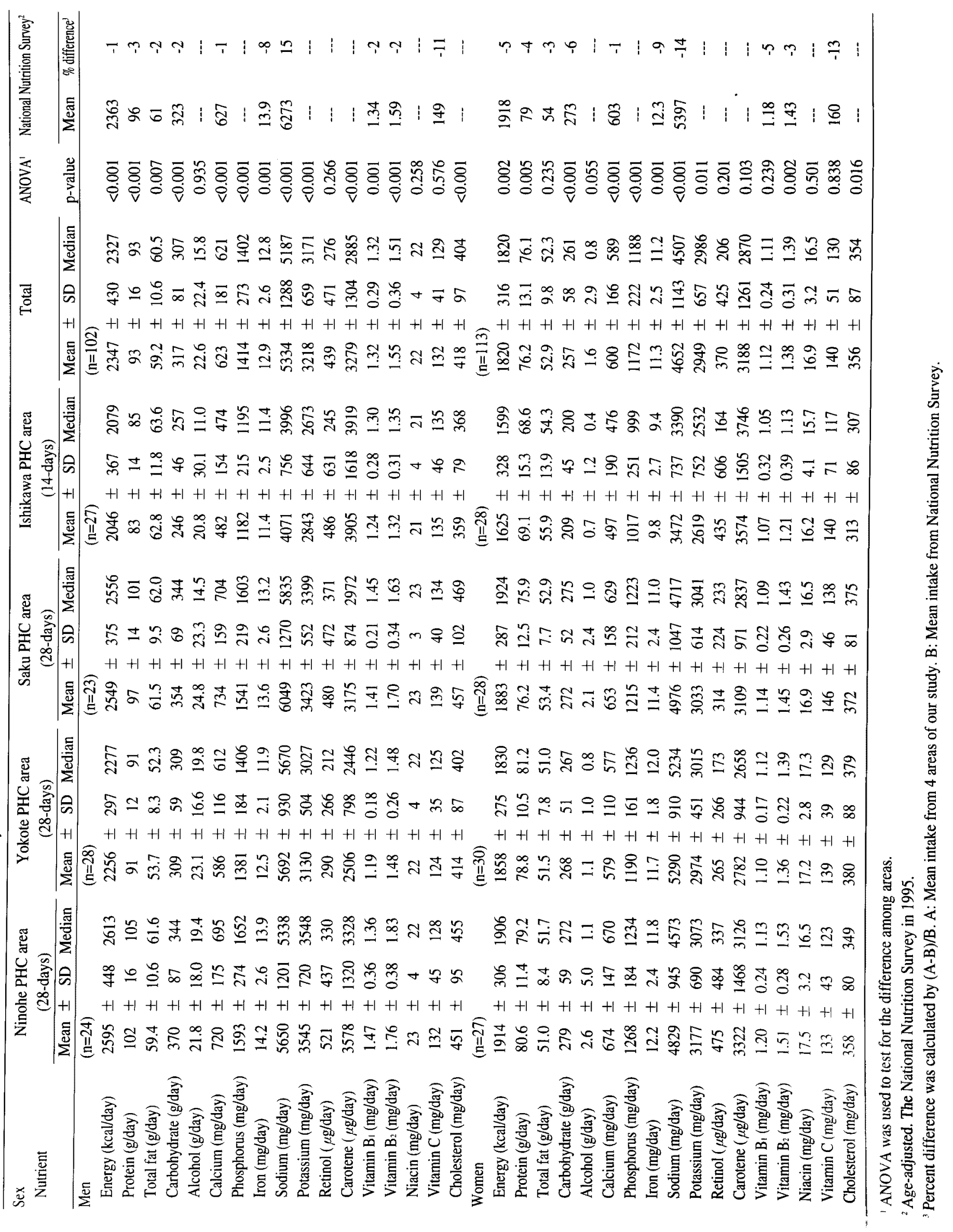


Table 2. Nutrient intakes (energy density) assessed with DR by area

\begin{tabular}{|c|c|c|c|c|c|c|c|c|c|c|c|c|c|c|c|c|c|c|c|c|c|}
\hline \multirow[t]{2}{*}{$\begin{array}{l}\text { Sex } \\
\text { Nutrient }\end{array}$} & \multicolumn{4}{|c|}{$\begin{array}{c}\text { Ninohe PHC area } \\
\text { (28-days) }\end{array}$} & \multicolumn{4}{|c|}{$\begin{array}{c}\text { Ninohe PHC area } \\
\text { (28-days) }\end{array}$} & \multicolumn{4}{|c|}{$\begin{array}{l}\text { Saku PHC area } \\
\text { (28-days) }\end{array}$} & \multicolumn{4}{|c|}{$\begin{array}{c}\text { Ishikawa PHC area } \\
\text { (14-days) }\end{array}$} & \multicolumn{4}{|c|}{ Total } & \multirow{2}{*}{$\begin{array}{l}\text { ANOVA }^{1} \\
\text { p-value }\end{array}$} \\
\hline & Mean & \pm & $\mathrm{SD}$ & Median & Mean & \pm & SD & Median & Mean & \pm & $\mathrm{SD}$ & Median & Mean & \pm & $\mathrm{SD}$ & Median & Mean & \pm & SD & $\overline{\text { Median }}$ & \\
\hline Men & $(n=24)$ & & & & $(n=28)$ & & & & $(n=23)$ & & & & $(n=27)$ & & & & $(n=102)$ & & & & \\
\hline Protein (\%E) & 15.8 & \pm & 1.7 & 15.7 & 16.2 & \pm & 1.5 & 16.1 & 15.3 & \pm & 1.3 & 15.1 & 16.4 & \pm & 1.7 & 16.8 & 16.0 & \pm & 1.6 & 15.9 & 0.080 \\
\hline Total fat $(\% \mathrm{E})$ & 20.9 & \pm & 3.4 & 20.6 & 21.5 & \pm & 2.7 & 21.2 & 21.9 & \pm & 3.0 & 21.3 & 27.8 & \pm & 4.3 & 27.9 & 23.1 & \pm & 4.4 & 22.3 & $<0.001$ \\
\hline Carbohydrate (\%E) & 56.6 & \pm & 5.3 & 56.8 & 54.5 & \pm & 5.3 & 54.5 & 55.7 & \pm & 6.5 & 56.8 & 48.4 & \pm & 6.7 & 47.7 & 53.6 & \pm & 6.7 & 54.0 & $<0.001$ \\
\hline Alcohol $(\% \mathrm{E})$ & 5.9 & \pm & 5.0 & 5.1 & 7.3 & \pm & 5.1 & 6.8 & 6.5 & \pm & 5.9 & 4.7 & 6.4 & \pm & 7.7 & 4.0 & 6.6 & \pm & 6.0 & 5.0 & 0.889 \\
\hline Calcium (mg/1000 kcal) & 280 & \pm & 63 & 275 & 260 & \pm & 45 & 259 & 290 & \pm & 58 & 286 & 236 & \pm & 65 & 231 & 265 & \pm & 61 & 262 & 0.007 \\
\hline Phosphorus (mg/1000 kcal) & 617 & \pm & 67 & 610 & 614 & \pm & 49 & 600 & 607 & \pm & 52 & 598 & 580 & \pm & 61 & 588 & 604 & \pm & 58 & 597 & 0.091 \\
\hline Iron $(\mathrm{mg} / 1000 \mathrm{kcal})$ & 5.5 & \pm & 0.8 & 5.5 & 5.6 & \pm & 0.7 & 5.5 & 5.3 & \pm & 0.7 & 5.3 & 5.6 & \pm & 1.0 & 5.4 & 5.5 & \pm & 0.8 & 5.4 & 0.616 \\
\hline Sodium $(\mathrm{mg} / 1000 \mathrm{kcal})$ & 2192 & \pm & 377 & 2203 & 2535 & \pm & 349 & 2430 & 2387 & \pm & 427 & 2403 & 2022 & \pm & 362 & 2013 & 2285 & \pm & 423 & 2328 & $<0.001$ \\
\hline Potassium (mg/1000 kcal) & 1381 & \pm & 251 & 1343 & 1389 & \pm & 151 & 1378 & 1349 & \pm & 156 & 1336 & 1396 & \pm & 232 & 1381 & 1380 & \pm & 200 & 1356 & 0.855 \\
\hline Retinol $(\mu \mathrm{g} / 1000 \mathrm{kcal})$ & 200 & \pm & 154 & 143 & 130 & \pm & 118 & 88 & 186 & \pm & 165 & 133 & 237 & \pm & 306 & 99 & 187 & \pm & 202 & 112 & 0.263 \\
\hline Carotene $(\mu \mathrm{g} / 1000 \mathrm{kcal})$ & 1415 & \pm & 543 & 1396 & 1116 & \pm & 322 & 1110 & 1253 & \pm & 321 & 1215 & 1916 & \pm & 712 & 1791 & 1429 & \pm & 588 & 1270 & $<0.001$ \\
\hline Vitamin $B_{1}(\mathrm{mg} / 1000 \mathrm{kcal})$ & 0.57 & \pm & 0.11 & 0.54 & 0.53 & \pm & 0.05 & 0.53 & 0.56 & \pm & 0.06 & 0.57 & 0.61 & \pm & 0.11 & 0.58 & 0.57 & \pm & 0.09 & 0.55 & 0.009 \\
\hline Vitamin $B_{2}(\mathrm{mg} / 1000 \mathrm{kcal})$ & 0.69 & \pm & 0.14 & 0.68 & 0.66 & \pm & 0.09 & 0.64 & 0.67 & \pm & 0.11 & 0.66 & 0.65 & \pm & 0.10 & 0.62 & 0.66 & \pm & 0.11 & 0.65 & 0.598 \\
\hline Niacin $(\mathrm{mg} / 1000 \mathrm{kcal})$ & 8.8 & \pm & 1.3 & 8.9 & 9.6 & \pm & 1.3 & 9.5 & 8.9 & \pm & 0.9 & 8.7 & 10.2 & \pm & 1.2 & 10.2 & 9.4 & \pm & 1.3 & 9.2 & $<0.001$ \\
\hline Vitamin C (mg/1000 kcal) & 51.4 & \pm & 16.3 & 52.8 & 54.7 & \pm & 13.0 & 51.0 & 54.7 & \pm & 12.6 & 52.4 & 67.1 & \pm & 22.7 & 63.3 & 57.2 & \pm & 17.6 & 54.0 & 0.006 \\
\hline $\begin{array}{l}\text { Cholesterol (mg/1000 kcal) } \\
\text { Women }\end{array}$ & $\begin{array}{c}176 \\
(n=27)\end{array}$ & \pm & 36 & 178 & $\begin{array}{c}184 \\
(n=30)\end{array}$ & \pm & 36 & 172 & $\begin{array}{c}181 \\
(n=28)\end{array}$ & \pm & 43 & 175 & $\begin{array}{c}177 \\
(\mathrm{n}=28)\end{array}$ & \pm & 32 & 176 & $\begin{array}{c}180 \\
(n=113)\end{array}$ & \pm & 36 & 175 & 0.836 \\
\hline Protein $(\% \mathbf{E})$ & 17.0 & \pm & 1.7 & 17.2 & 17.0 & \pm & 1.2 & 17.0 & 16.2 & \pm & 1.4 & 16.6 & 17.0 & \pm & 1.5 & 17.0 & 16.8 & \pm & 1.5 & 16.9 & 0.111 \\
\hline Total fat (\%E) & 24.1 & \pm & 2.8 & 24.5 & 25.1 & \pm & 3.4 & 24.7 & 25.7 & \pm & 2.8 & 26.2 & 30.8 & \pm & 3.7 & 31.5 & 26.4 & \pm & 4.1 & 26.0 & $<0.001$ \\
\hline Carbohydrate (\%E) & 58.1 & \pm & 4.1 & 57.5 & 57.4 & \pm & 4.2 & 58.0 & 57.5 & \pm & 3.9 & 57.0 & 51.6 & \pm & 5.3 & 51.1 & 56.1 & \pm & 5.1 & 56.7 & $<0.001$ \\
\hline Alcohol $(\% \mathbf{E})$ & 1.0 & \pm & 2.0 & 0.4 & 0.4 & \pm & 0.3 & 0.3 & 0.7 & \pm & 0.8 & 0.4 & 0.3 & \pm & 0.5 & 0.1 & 0.6 & \pm & 1.1 & 0.3 & 0.087 \\
\hline Calcium (mg/1000 kcal) & 354 & \pm & 61 & 349 & 316 & \pm & 65 & 316 & 345 & \pm & 54 & 333 & 302 & \pm & 89 & 279 & 329 & \pm & 71 & 326 & 0.018 \\
\hline Phosphorus (mg/1000 kcal) & 666 & \pm & 58 & 668 & 643 & \pm & 53 & 644 & 645 & \pm & 50 & 647 & 623 & \pm & 77 & 612 & 644 & \pm & 61 & 645 & 0.079 \\
\hline Iron $(\mathrm{mg} / 1000 \mathrm{kcal})$ & 6.4 & \pm & 1.0 & 6.4 & 6.3 & \pm & 0.5 & 6.3 & 6.0 & \pm & 0.7 & 6.0 & 6.0 & \pm & 0.9 & 5.8 & 6.2 & \pm & 0.8 & 6.2 & 0.139 \\
\hline Sodium (mg/1000 kcal) & 2538 & \pm & 388 & 2465 & 2861 & \pm & 406 & 2741 & 2639 & \pm & 353 & 2603 & 2161 & \pm & 342 & 2166 & 2555 & \pm & 449 & 2537 & $<0.001$ \\
\hline Potassium (mg/1000 kcal) & 1663 & \pm & 259 & 1661 & 1614 & \pm & 236 & 1595 & 1603 & \pm & 130 & 1607 & 1601 & \pm & 256 & 1616 & 1620 & \pm & 224 & 1608 & 0.718 \\
\hline Retinol ( $\mu \mathrm{g} / 1000 \mathrm{kcal})$ & 257 & \pm & 281 & 199 & 141 & \pm & 133 & 96 & 172 & \pm & 136 & 120 & 244 & \pm & 304 & 106 & 202 & \pm & 228 & 114 & 0.163 \\
\hline Carotene $(\mu \mathrm{g} / 1000 \mathrm{kcal})$ & 1750 & \pm & 747 & 1674 & 1524 & \pm & 558 & 1407 & 1632 & \pm & 343 & 1565 & 2177 & \pm & 714 & 2077 & 1767 & \pm & 651 & 1699 & 0.001 \\
\hline Vitamin $B_{1}(\mathrm{mg} / 1000 \mathrm{kcal})$ & 0.63 & \pm & 0.12 & 0.62 & 0.59 & \pm & 0.07 & 0.58 & 0.60 & \pm & 0.06 & 0.60 & 0.65 & \pm & 0.11 & 0.64 & 0.62 & \pm & 0.09 & 0.61 & 0.063 \\
\hline Vitamin $B_{2}(m g / 1000 \mathrm{kcal})$ & 0.79 & \pm & 0.12 & 0.77 & 0.74 & \pm & 0.11 & 0.73 & 0.77 & \pm & 0.09 & 0.76 & 0.74 & \pm & 0.15 & 0.72 & 0.76 & \pm & 0.12 & 0.75 & 0.230 \\
\hline Niacin $(\mathrm{mg} / 1000 \mathrm{kcal})$ & 9.2 & \pm & 1.3 & 8.9 & 9.3 & \pm & 1.0 & 9.3 & 9.0 & \pm & 1.0 & 8.8 & 9.9 & \pm & 1.3 & 10.0 & 9.4 & \pm & 1.2 & 9.3 & 0.021 \\
\hline Vitamin C (mg/1000 kcal) & 69.3 & \pm & 18.2 & 65.7 & 75.4 & \pm & 21.2 & 73.5 & 76.9 & \pm & 16.6 & 75.5 & 84.2 & \pm & 33.5 & 77.6 & 76.5 & \pm & 23.6 & 73.3 & 0.134 \\
\hline Cholesterol (mg/1000 kcal) & 189 & \pm & 40 & 183 & 205 & \pm & 41 & 201 & 200 & \pm & 42 & 204 & 193 & \pm & 40 & 199 & 197 & \pm & 41 & 201 & 0.473 \\
\hline
\end{tabular}

1 ANOVA was used to test for the difference among areas. 


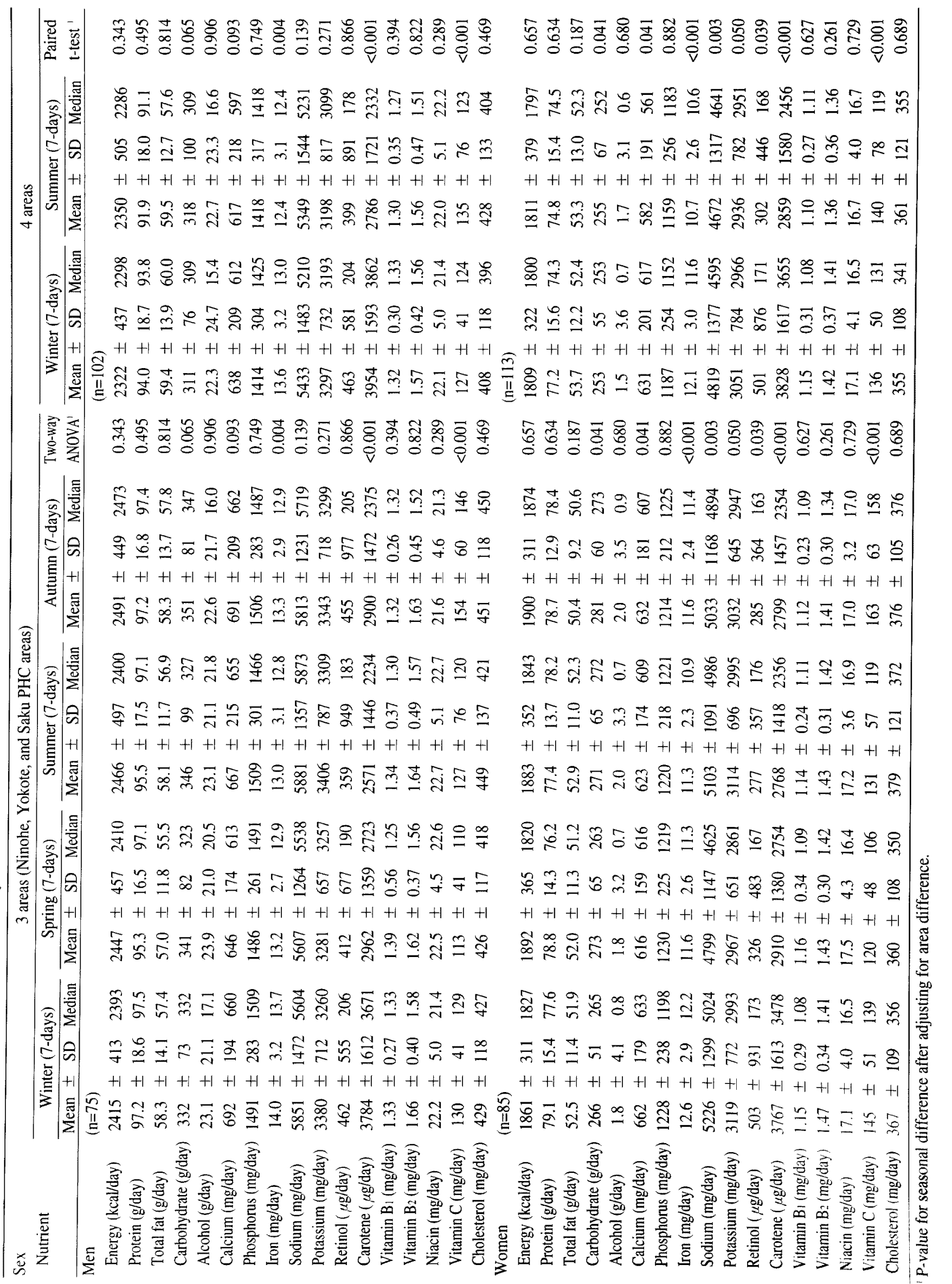




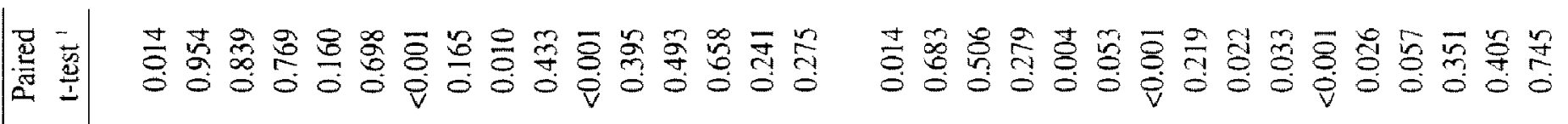

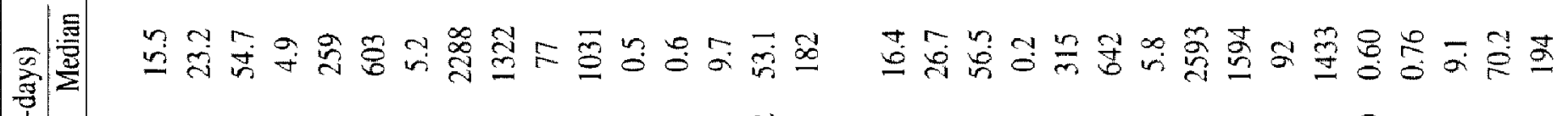

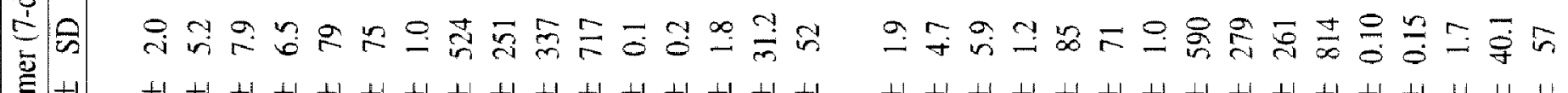
琒

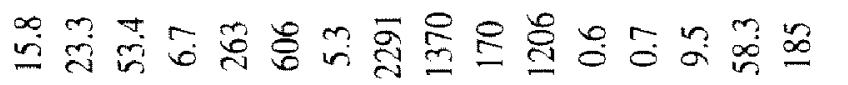
$+1+H+H+1+H+H+1+H+H+1+H+H$

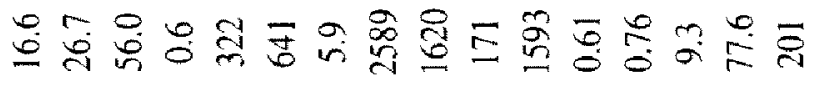

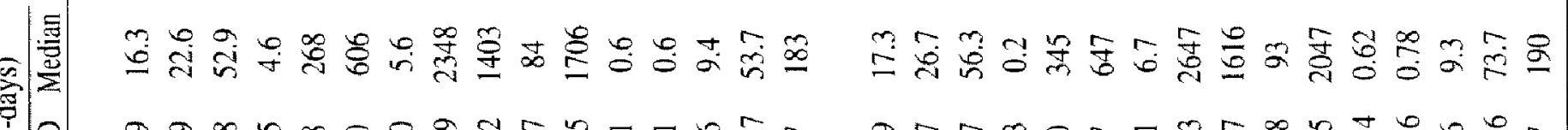

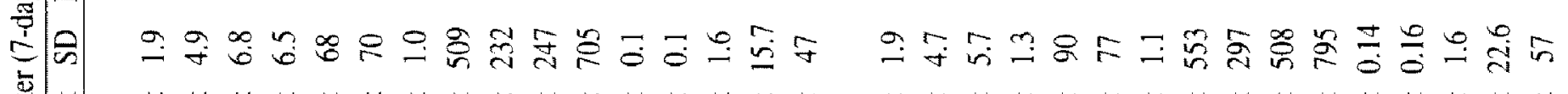

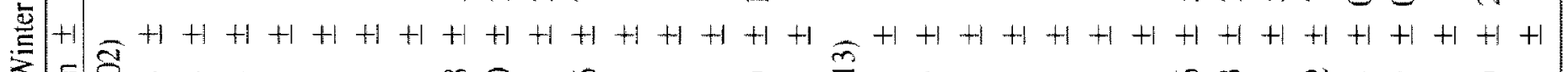

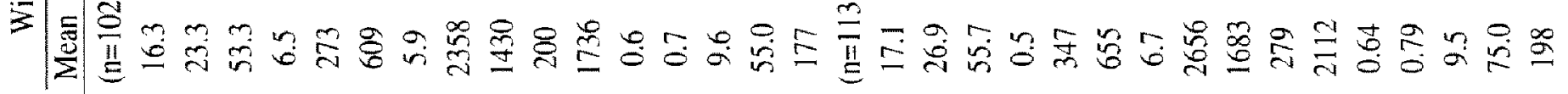
|

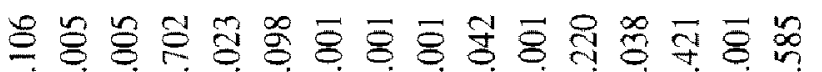

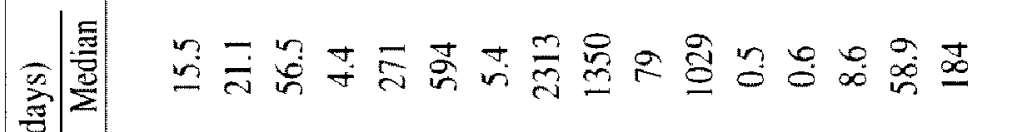

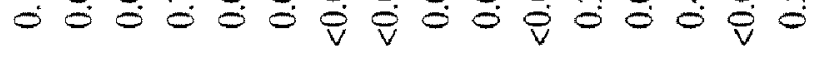

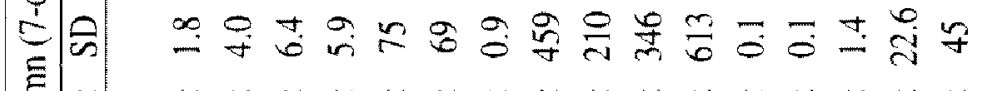

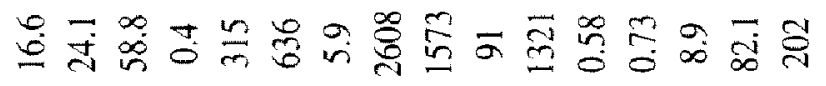

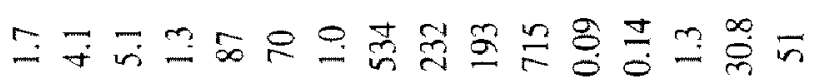
$+1+1+1+1+1+1+1+1+1+1+1+1+1+1+1+1$ $+1+1+1+1+1+1+1+1+1+1+1+1+1+1$

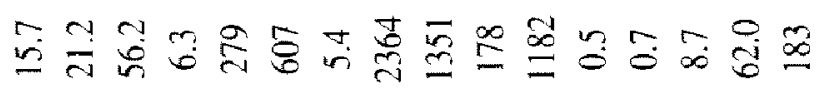

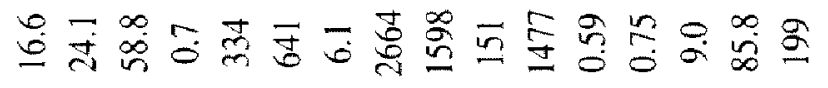

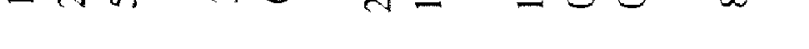

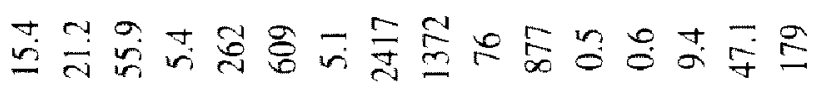

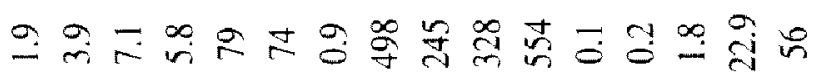

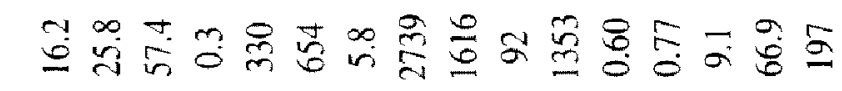
$\mathrm{H}+\mathrm{H}+\mathrm{H}+\mathrm{H}+\mathrm{H}+\mathrm{H}+\mathrm{H}+\mathrm{H}+\mathrm{H}+\mathrm{H}+\mathrm{H}+\mathrm{H}+\mathrm{H}$

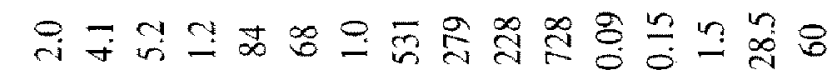

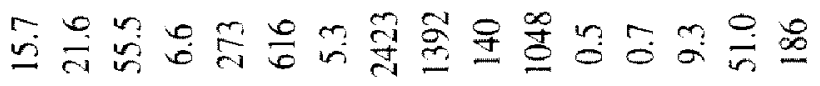
$+1+1+1+1+1+1+1+1+1+1+1+1+1+1+1+1$

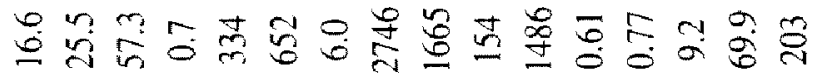

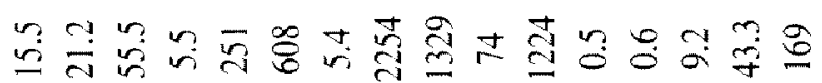

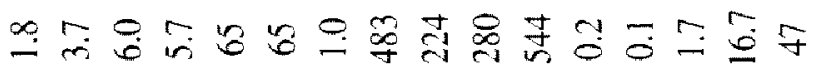
$+\mathrm{H}+\mathrm{H} H+\mathrm{H} H \mathrm{H}+\mathrm{H}+\mathrm{H}+\mathrm{H}+\mathrm{H}+\mathrm{H}+\mathrm{H} H$

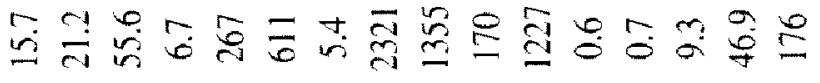

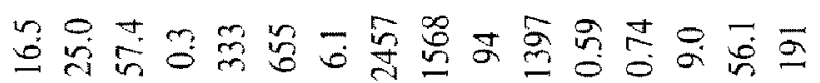

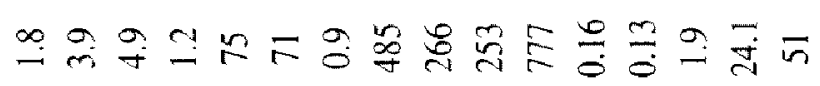
$\mathrm{H}+\mathrm{H}+\mathrm{H}+\mathrm{H}+\mathrm{H}+\mathrm{H}+\mathrm{H}+\mathrm{H}+\mathrm{H}+\mathrm{H}+\mathrm{H}+\mathrm{H}$

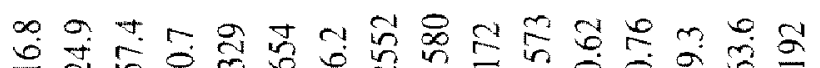

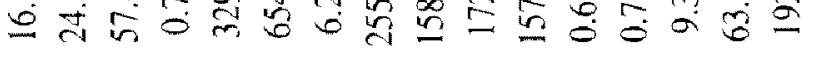

溇 응 9 웡

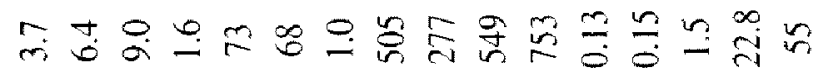
焉 $+1+1+1+1+1+1+1+1+1+1+1+1+1+1+1+1$ $+1+1+1+1+1+1+1+1+1+1+1+1+1+1+1$

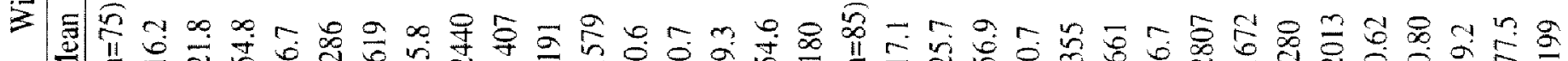

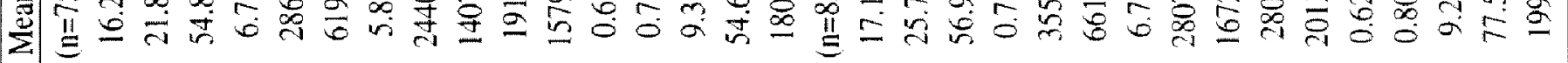

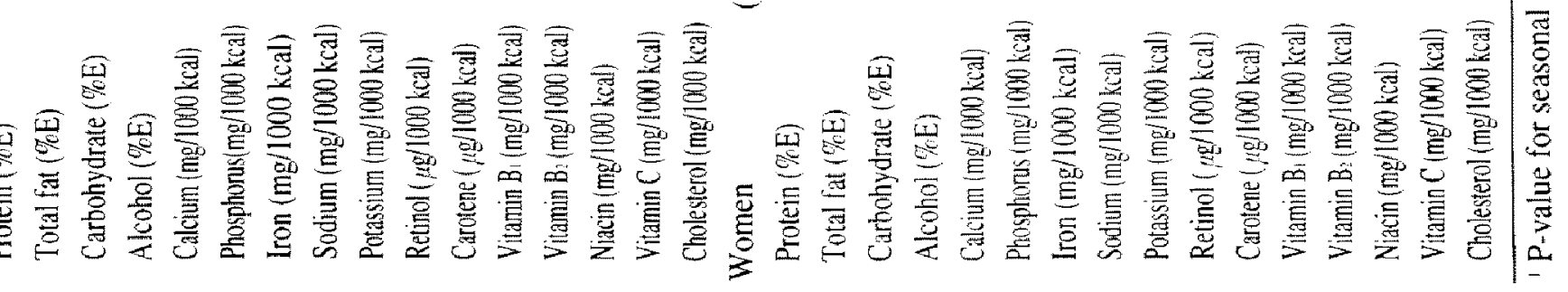




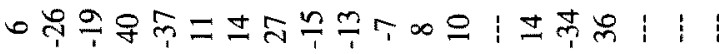

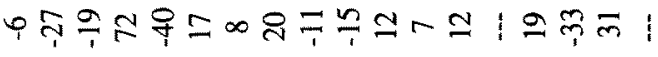

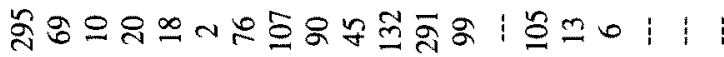

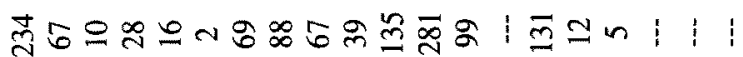

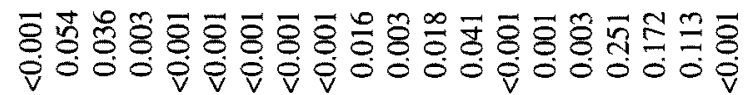

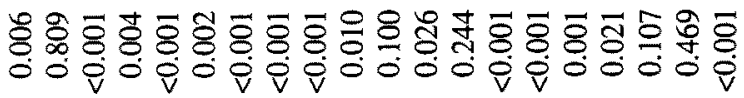

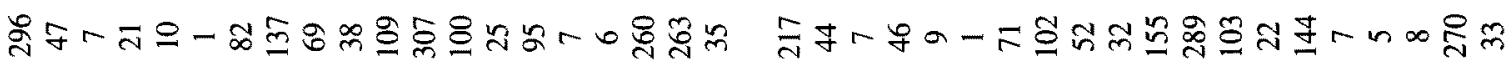

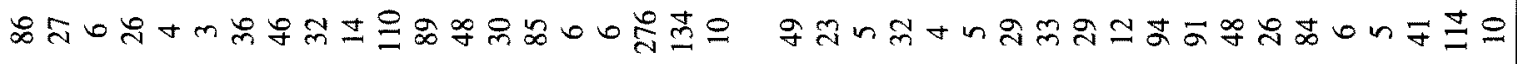

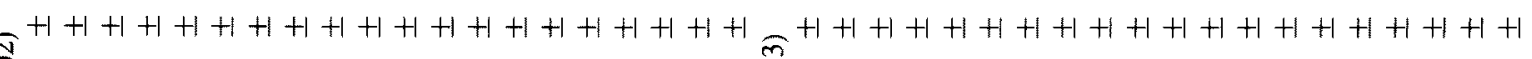
严向的只ニ

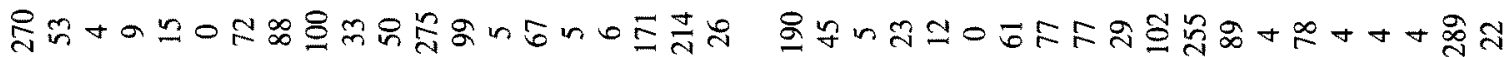

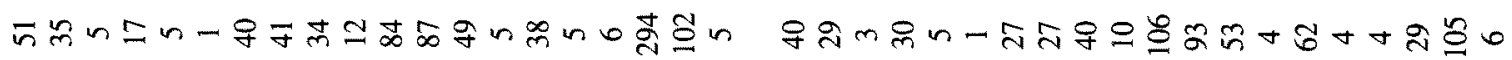
$\mathrm{H}+\mathrm{H}+\mathrm{H}+\mathrm{H}+\mathrm{H}+\mathrm{H}+\mathrm{H}+\mathrm{H}+\mathrm{H}+\mathrm{H}+\mathrm{H}+\mathrm{H}+\mathrm{H}+\mathrm{H}+\mathrm{H}+\mathrm{H} \quad \mathrm{H}+\mathrm{H}+\mathrm{H}+\mathrm{H}+\mathrm{H}+\mathrm{H}+\mathrm{H}+\mathrm{H}+\mathrm{H}+\mathrm{H}+\mathrm{H}+\mathrm{H}+\mathrm{H}+\mathrm{H}+\mathrm{H}+\mathrm{H}$

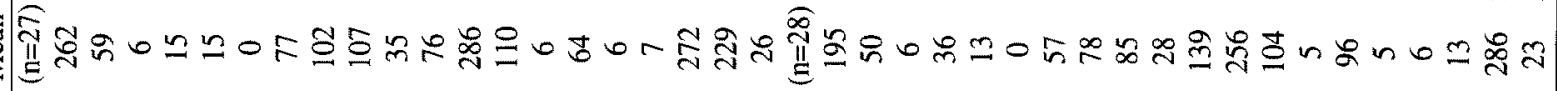

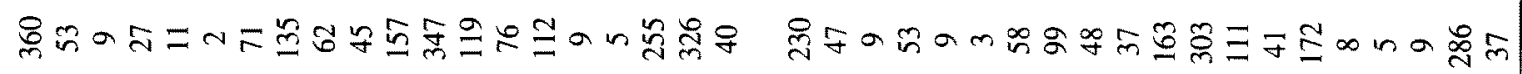

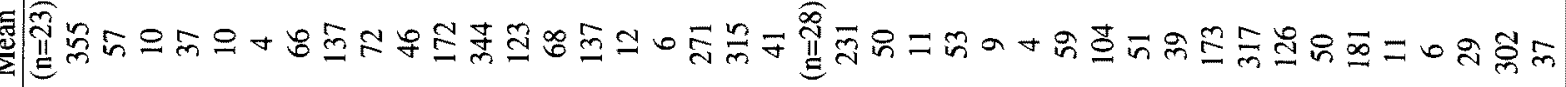

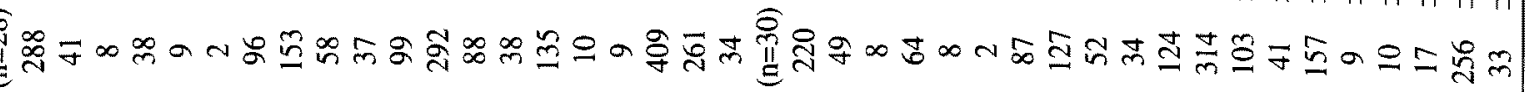

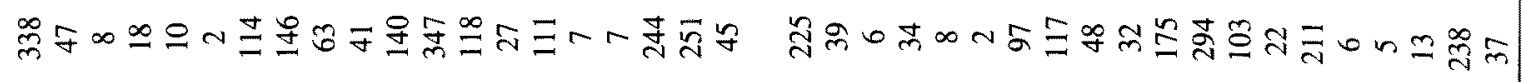

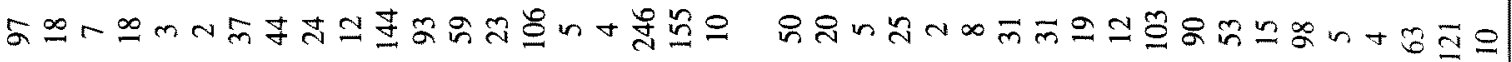
$H+H+1+H+1+1+1+1+1+1+1+1+1+1+1+1+1+1+1 \quad+1+1+1+1+1+1+1+1+1+1+1+1+1+1+1+1+1+1+1+1$ 


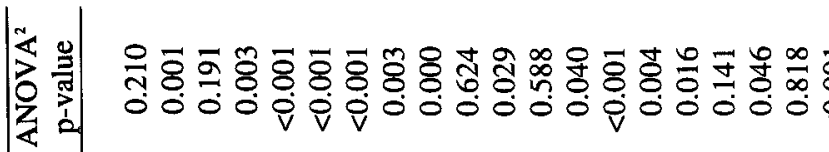

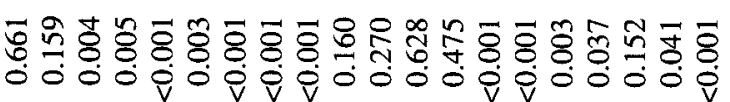

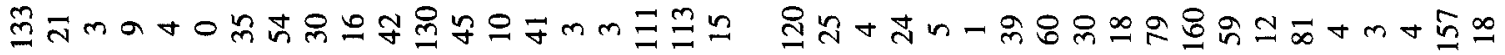

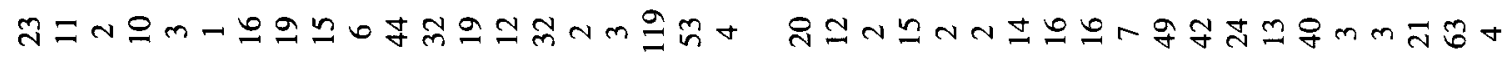
$+1+1+1+H+1+H+1+1+1+1+1+1+1+1+1+1+1 \quad+1+1+1+1+1+1+1+1+1+1+1+1+1+1+1+1+1+1$

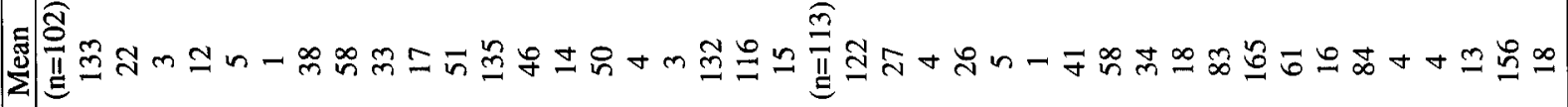

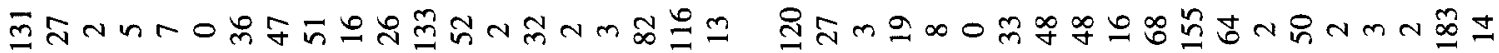
กีะกmо $+1+1+1+1+1+1+1+1+1+1+1+1+1+1+1+1+1+1+1+1 \quad+1+1+1+1+1+1+1+1+1+1+1+1+1+1+1+1+1+1+1+1$

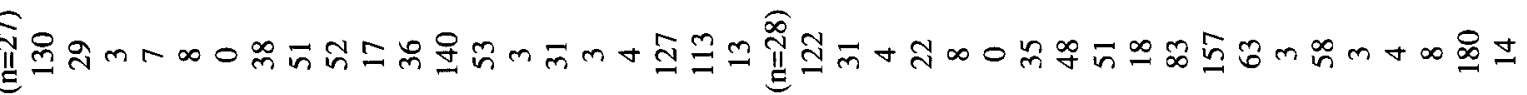

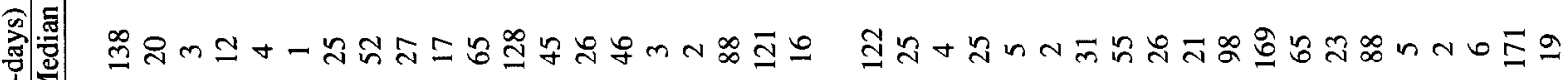
กี

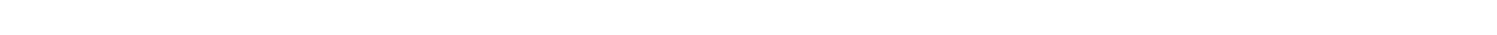

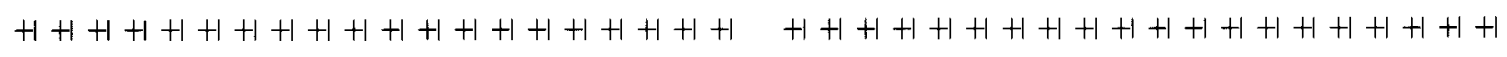

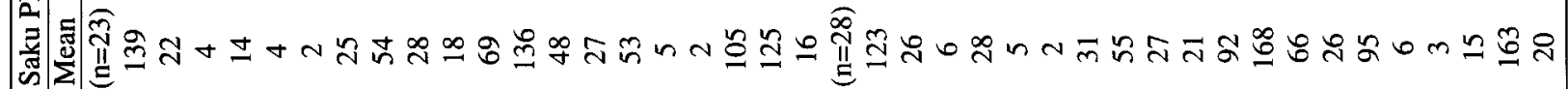

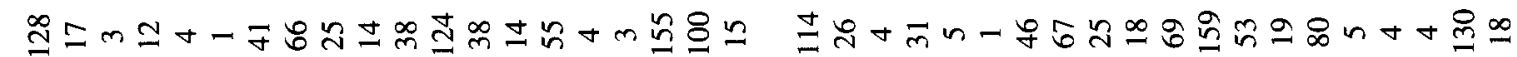

$H+1+1+1+1+1+1+1+1+1+1+1+1+1+1+1+1+1$

$+\mathrm{H}+\mathrm{H}+\mathrm{H}+\mathrm{H}+\mathrm{H}+\mathrm{H}+\mathrm{H}+\mathrm{H}+\mathrm{H}+\mathrm{H}+\mathrm{H}+\mathrm{H}+\mathrm{H}+\mathrm{H}$

$\infty m \simeq \forall-m \infty$

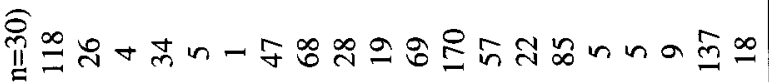

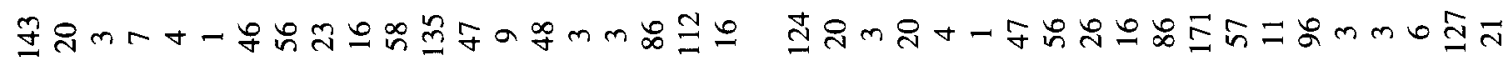

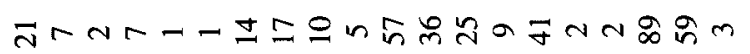

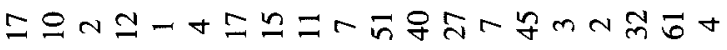

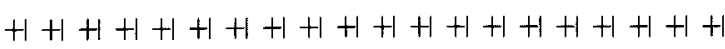
$+\mathrm{H}+\mathrm{H}+\mathrm{H}+\mathrm{H}+\mathrm{H}+\mathrm{H}+\mathrm{H}+\mathrm{H}+\mathrm{H}+\mathrm{H}+\mathrm{H}+\mathrm{H}+\mathrm{H}+\mathrm{H}+\mathrm{H}+\mathrm{H}$

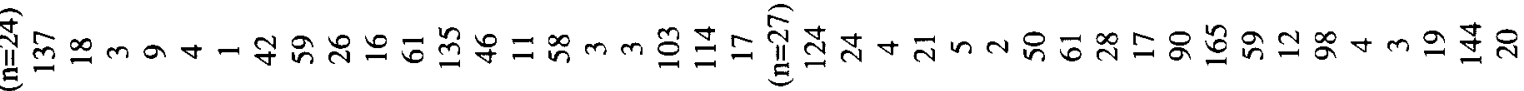

(2)

产

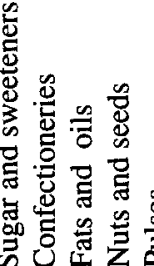

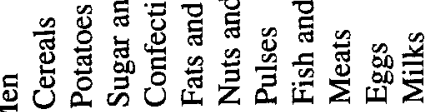
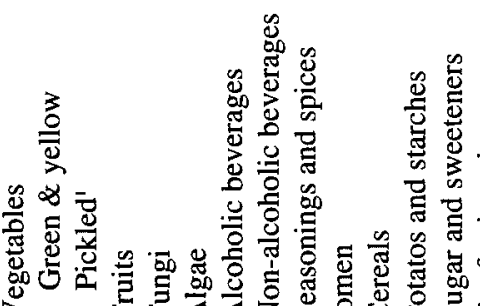

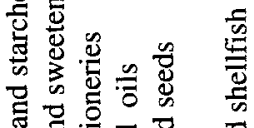

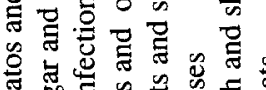

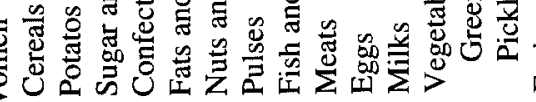

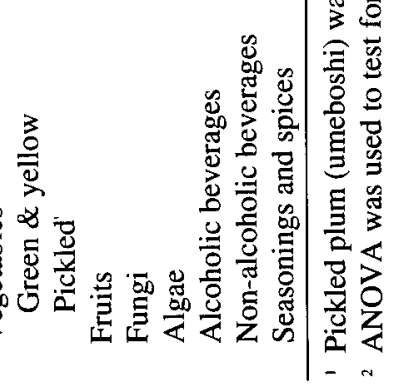


|

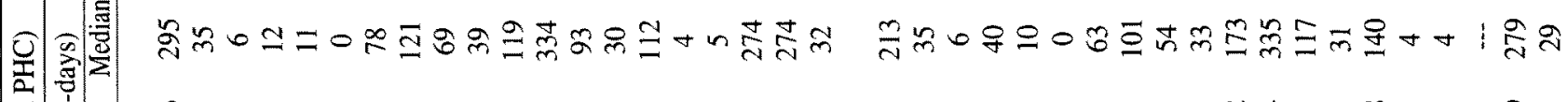

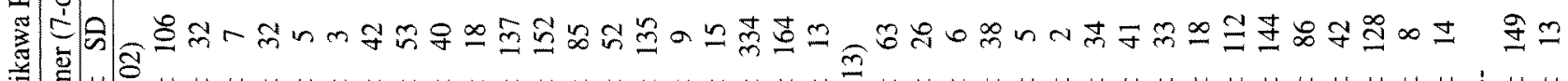
娄 马)

닐

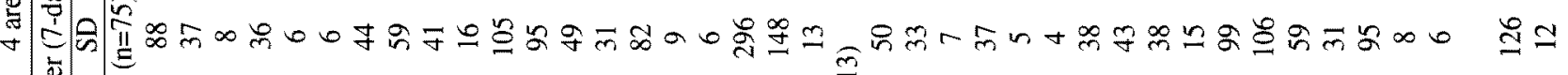
总 $+1+1+1+1+1+1+1+1+1+1+1+1+1+1+1+1+1+1+\frac{\varrho}{=}+1+1+1+1+1+1+1+1+1+1+1+1+1+1+1+1+1 \mid+1+1$

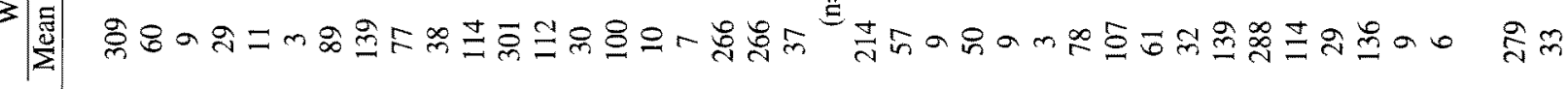

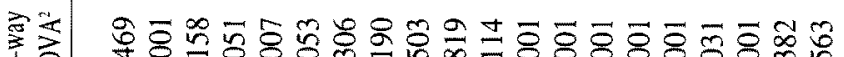

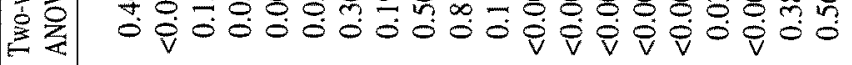

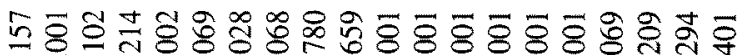

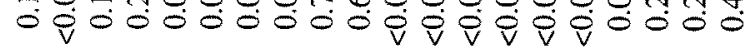

舒

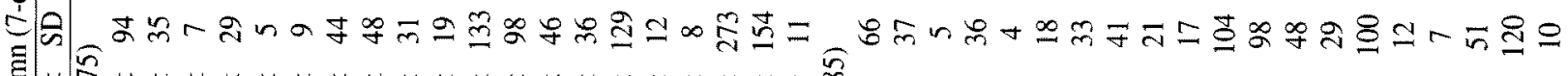

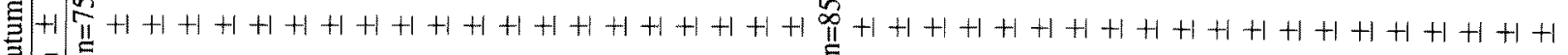

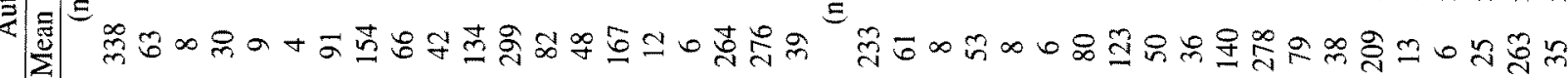

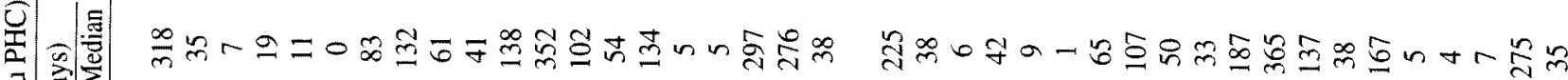

으에요 $\operatorname{los}_{0}$

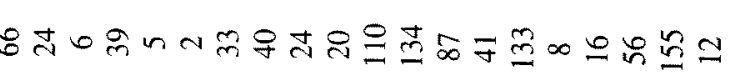
il $\mathrm{H}+\mathrm{H}+\mathrm{H}+\mathrm{H}+\mathrm{H}+\mathrm{H}+\mathrm{H}+\mathrm{H}+\mathrm{H}+\mathrm{H}+\mathrm{H}+\mathrm{H}+\mathrm{H}+\mathrm{H}$ m

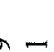
$\infty \underset{3}{2}$ so

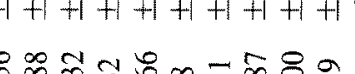

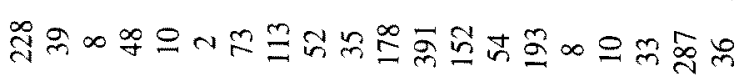

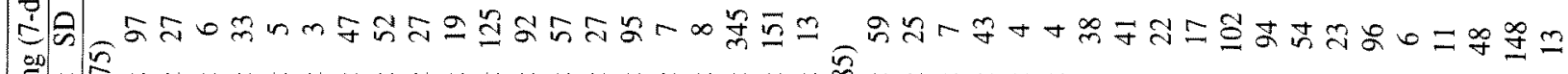
.

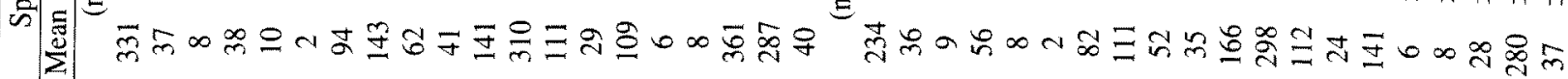

矛

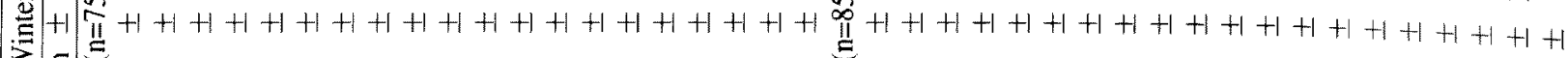

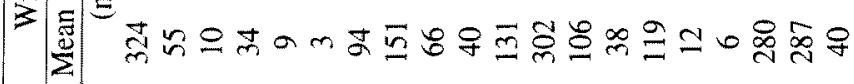

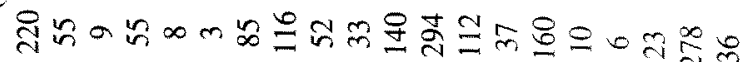

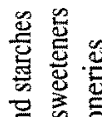

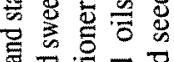
क

产

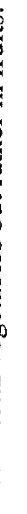




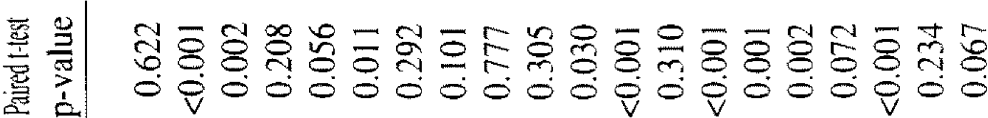

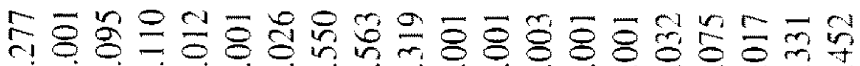

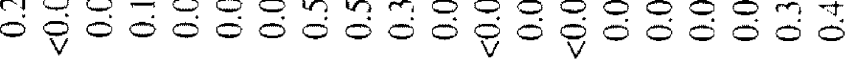

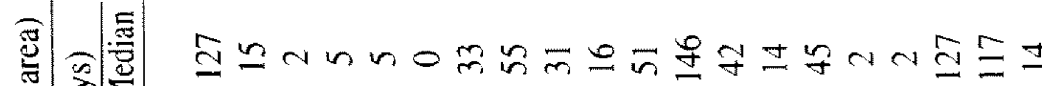

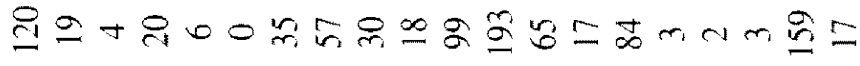

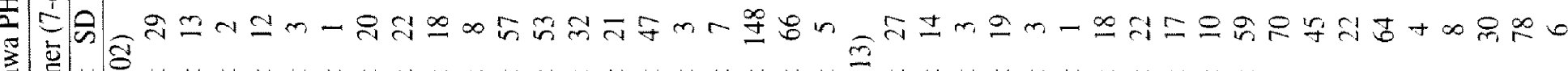

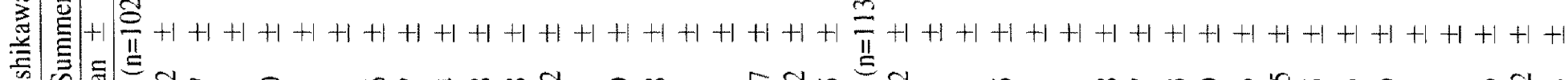

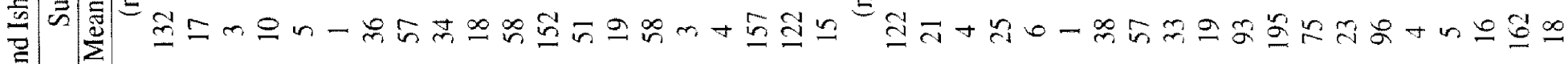

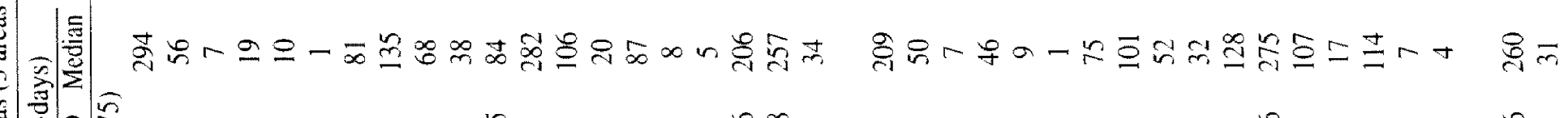

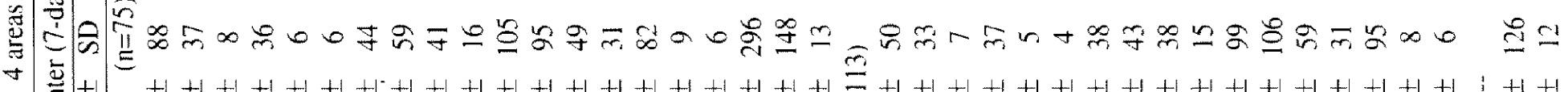

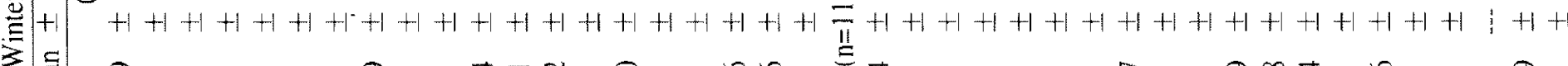

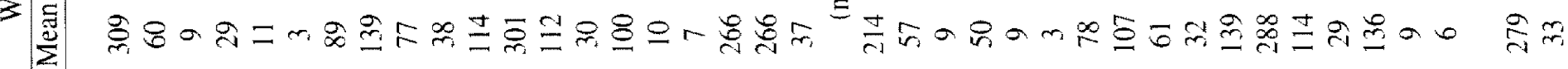

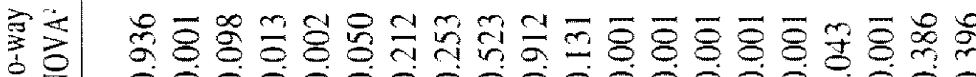

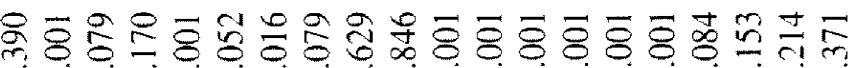
妾

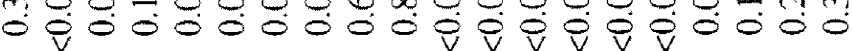

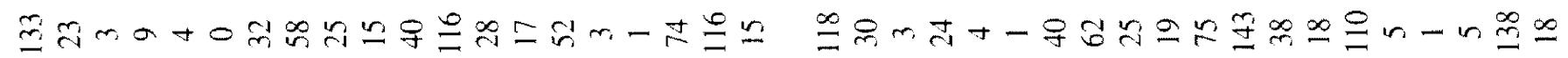

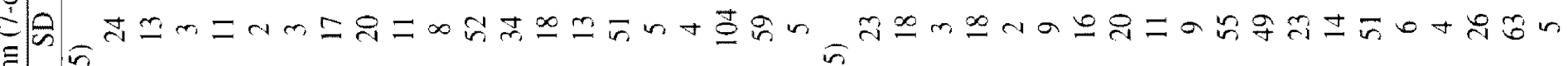
+1 III $+1+1+1+1+1+1+1+1+1+1+1+1+1+1+1+1+1+1+1$ 竞 $+1+1+1+1+1+1+1+1+1+1+1+1+1+1+1+1+1+1+1+1$

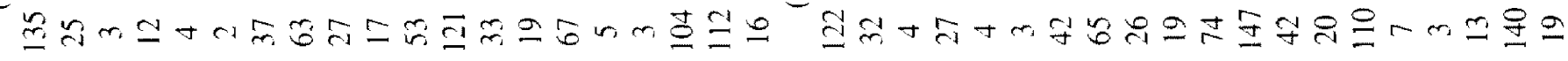

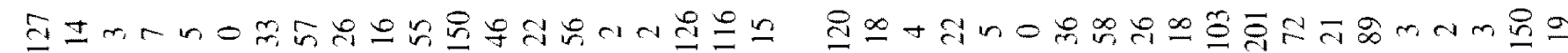

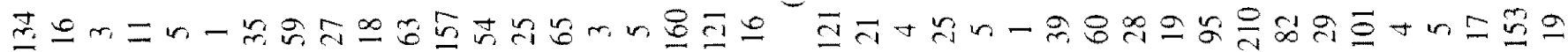

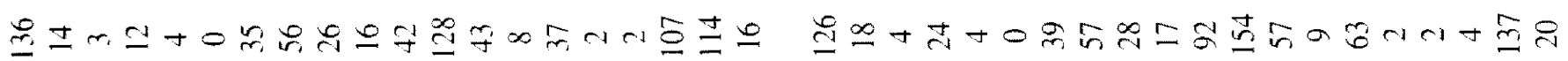

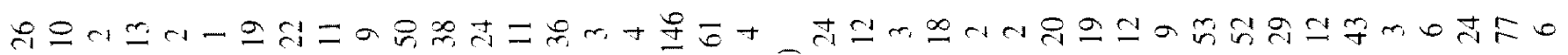

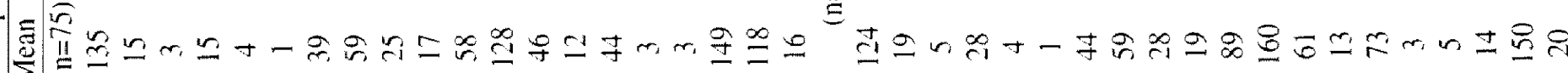

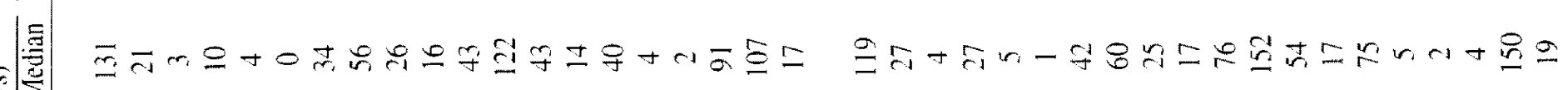

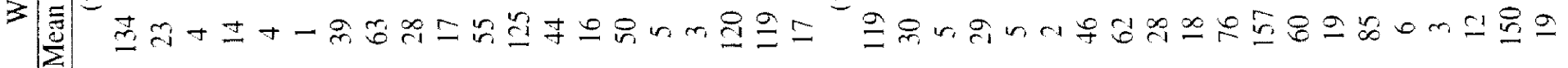


Table 9. Cumulative \% contribution of the top 20 foods for energy assessed by DR

\begin{tabular}{|c|c|c|c|}
\hline Food code ${ }^{1}$ & Description' & $\mathrm{kcal} /$ day & $\begin{array}{c}\text { Cumulative }^{2} \\
\text { percent }\end{array}$ \\
\hline \multicolumn{4}{|l|}{ Men $(n=102)$} \\
\hline $1-41 d$ & Rice (Paddy rice)/Grains/Well-milled rice & 816.5 & 34.8 \\
\hline 5-15 & Fats and oils/Vegetable oils/Vegetable oil, mixed & 96.9 & 38.9 \\
\hline $16-2 \mathrm{a}$ & Fermented alcoholic beverages/Beer/Pale & 83.2 & 42.5 \\
\hline $16-1 b$ & Fermented alcoholic beverages/Sake/First class & 65.2 & 45.3 \\
\hline $10-5 a$ & Chicken egg/Whole egg/fresh & 61.8 & 47.9 \\
\hline $11-2$ & Liquid milk/Ordinary liquid milk & 61.5 & 50.5 \\
\hline $16-4 b$ & Distilled alcoholic beverages/Shochu/25\% alcohol & 36.5 & 52.1 \\
\hline $7-32 \mathrm{c}$ & Miso/Rice-koji Miso/Dark yellow type & 36.2 & 53.6 \\
\hline $7-21 \mathrm{a}$ & Tofu and Abura-age/Tofu, soybean curd/Momen & 26.0 & 54.7 \\
\hline $1-26 a$ & Chinese noodles/Chinese noodle i.e., Lao Mien/Raw, dry form & 23.7 & 55.7 \\
\hline $1-13 a$ & Wheat (Breads)/White bread/On the market & 23.7 & 56.7 \\
\hline 9-70a & Pork and separable fat/Belly without separable fat/Large-type & 23.2 & 57.7 \\
\hline $3-4 a$ & Sugar/Soft sugar/White, superior & 22.1 & 58.7 \\
\hline $1-10 \mathrm{a}$ & Wheat flour/Soft flour/First grade & 21.3 & 59.6 \\
\hline $1-21 b$ & Japanese noodles/Udon/Boiled & 21.0 & 60.5 \\
\hline $2-11 \mathrm{a}$ & Potatoes/Tuber/Raw & 20.8 & 61.4 \\
\hline $8-150 \mathrm{a}$ & Tunas/Bluefin tuna, raw/Lean meat & 15.4 & 62.0 \\
\hline $8-84 a$ & Mackerel/Raw/Uncooked & 14.6 & 62.6 \\
\hline $1-24 b$ & Japanese noodles/Somen Hiyamugi/Boiled & 14.4 & 63.3 \\
\hline $13-88$ & Apples/Raw fruit & 14.3 & 63.9 \\
\hline \multicolumn{4}{|c|}{ Women $(n=113)$} \\
\hline $1-41 d$ & Rice (Paddy rice)/Grains/Well-milled rice & 523.7 & 28.8 \\
\hline $5-15$ & Fats and oils/Vegetable oils/Vegetable oil, mixed & 82.7 & 33.3 \\
\hline $11-2$ & Liquid milk/Ordinary liquid milk & 70.6 & 37.2 \\
\hline $10-5 a$ & Chicken egg/Whole egg/fresh & 51.7 & 40.0 \\
\hline $7-32 c$ & Miso/Rice-koji Miso/Dark yellow type & 30.9 & 41.7 \\
\hline $1-13 a$ & Wheat (Breads)/White bread/On the market & 29.0 & 43.3 \\
\hline $3-4 a$ & Sugar/Soft sugar/White, superior & 22.2 & 44.6 \\
\hline 9-70a & Pork and separable fat/Belly without separable fat/Large-type & 21.7 & 45.7 \\
\hline $7-21 \mathrm{a}$ & Tofu and Abura-age/Tofu, soybean curd/Momen & 21.3 & 46.9 \\
\hline $1-10 \mathrm{a}$ & Wheat flour/Soft flour/First grade & 19.6 & 48.0 \\
\hline $2-11 a$ & Potatoes/Tuber/Raw & 19.0 & 49.0 \\
\hline $1-26 a$ & Chinese noodles/Chinese noodle i.e., Lao Mien/Raw, dry form & 18.8 & 50.1 \\
\hline $1-21 b$ & Japanese noodles/Udon/Boiled & 18.8 & 51.1 \\
\hline $13-88$ & Apples/Raw fruit & 17.8 & 52.1 \\
\hline $17-10 \mathrm{a}$ & Seasonings/Mayonnaise/Whole egg type & 15.7 & 52.9 \\
\hline $4-24 \mathrm{e}$ & Japanese-style undried and semi-dried confectioneries/Manju/Mushi & 13.5 & 53.7 \\
\hline $4-46$ & Western-style undried and semi-dried confectioneries/Shortcake & 12.7 & 54.4 \\
\hline $7-29$ & Natto, fermented soybeans/Itohiki-natto & 12.5 & 55.1 \\
\hline $8-95 a$ & Pacific saury/Raw/Uncooked & 11.4 & 55.7 \\
\hline $1-24 b$ & Japanese noodles/Somen Hiyamugi/Boiled & 11.1 & 56.3 \\
\hline
\end{tabular}

1 Food codes and descriptions correspond to those of the Standard Tables of Food Composition, 4th revised edition in Japan by Science and Technology Agency.

${ }^{2}$ Data on subjects in Ishikawa PHC (14-day data) were counted twice for 28-day data. 
Table 10. Cumulative $\%$ contribution of the top 20 foods for protein assessed by DR

\begin{tabular}{|c|c|c|c|}
\hline Food code ${ }^{1}$ & Description $^{1}$ & g/day & $\begin{array}{c}\text { Cumulative }^{2} \\
\text { percent }\end{array}$ \\
\hline \multicolumn{4}{|l|}{ Men $(n=102)$} \\
\hline $1-41 d$ & Rice (Paddy rice)/Grains/Well-milled rice & 15.6 & 16.8 \\
\hline $10-5 a$ & Chicken egg/Whole egg/fresh & 4.7 & 21.8 \\
\hline $8-150 \mathrm{a}$ & Tunas/Bluefin tuna, raw/Lean meat & 3.3 & 25.4 \\
\hline $11-2$ & Liquid milk/Ordinary liquid milk & 3.0 & 28.6 \\
\hline $7-32 c$ & Miso/Rice-koji Miso/Dark yellow type & 2.6 & 31.4 \\
\hline $7-21 \mathrm{a}$ & Tofu and Abura-age/Tofu, soybean curd & 2.3 & 33.8 \\
\hline $8-206 a$ & Squid and cuttlefish/Raw/Uncooked & 1.8 & 35.8 \\
\hline $17-3 a$ & Seasonings/Shoyu, soy-sauce/Koikuchi & 1.5 & 37.4 \\
\hline $8-78 \mathrm{a}$ & Salmons/Mild salted chum salmon & 1.4 & 38.9 \\
\hline $9-47 b$ & Chicken and separable fat/Breast & 1.3 & 40.2 \\
\hline $8-84 a$ & Mackerel/Raw/Uncooked & 1.2 & 41.6 \\
\hline $8-95 a$ & Pacific saury/Raw/Uncooked & 1.2 & 42.8 \\
\hline $7-29$ & Natto, fermented soybeans/Itohiki-natto & 1.1 & 44.0 \\
\hline $7-22$ & Tofu and Abura-age/Okinawa-tofu & 1.1 & 45.2 \\
\hline $9-68 \mathrm{a}$ & Pork/Loin, separable lean & 1.0 & 46.3 \\
\hline $8-77$ & Salmons/Chum salmon, raw & 1.0 & 47.4 \\
\hline $9-70 \mathrm{a}$ & Pork/Belly without separable fat & 1.0 & 48.5 \\
\hline $8-218$ & Prawns/Spiny lobster, raw & 0.9 & 49.4 \\
\hline $9-49 b$ & Chicken and separable fat/Thigh/Broiler & 0.9 & 50.4 \\
\hline $9-72 a$ & Pork/Inside ham, separable lean & 0.9 & 51.4 \\
\hline \multicolumn{4}{|c|}{ Women $(n=113)$} \\
\hline $1-41 d$ & Rice (Paddy rice)/Grains/Well-milled rice & 10.0 & 13.1 \\
\hline $10-5 \mathrm{a}$ & Chicken egg/Whole egg/fresh & 3.9 & 18.3 \\
\hline $11-2$ & Liquid milk/Ordinary liquid milk & 3.5 & 22.8 \\
\hline $7-32 c$ & Miso/Rice-koji Miso/Dark yellow type & 2.2 & 25.7 \\
\hline $8-150 a$ & Tunas/Bluefin tuna, raw/Lean meat & 2.1 & 28.4 \\
\hline $7-21 a$ & Tofu and Abura-age/Tofu, soybean curd & 1.9 & 30.9 \\
\hline $17-3 a$ & Seasonings/Shoyu, soy-sauce/Koikuchi & 1.3 & 32.6 \\
\hline $8-206 a$ & Squid and cuttlefish/Raw/Uncooked & 1.3 & 34.3 \\
\hline $8-78 a$ & Salmon/Mild salted chum salmon & 1.3 & 35.9 \\
\hline $7-29$ & Natto, fermented soybeans/Itohiki-natto & 1.0 & 37.3 \\
\hline $9-47 \mathrm{~b}$ & Chicken and separable fat/Breast & 1.0 & 38.6 \\
\hline $8-95 \mathrm{a}$ & Pacific saury/Raw/Uncooked & 1.0 & 39.9 \\
\hline $1-13 a$ & Wheat (Breads)/White bread/On the market & 0.9 & 41.1 \\
\hline 9-70a & Pork/Belly without separable fat & 0.9 & 42.3 \\
\hline $8-84 a$ & Mackerel/Raw/Uncooked & 0.9 & 43.5 \\
\hline $8-77$ & Salmon/Chum salmon, raw & 0.9 & 44.7 \\
\hline $9-49 b$ & Chicken and separable fat/Thigh/Broiler & 0.9 & 45.8 \\
\hline $7-22$ & Tofu and Abura-age/Okinawa-tofu & 0.8 & 46.9 \\
\hline $9-68 \mathrm{a}$ & Pork/Loin, separable lean & 0.8 & 47.9 \\
\hline $9-72 a$ & Pork/Inside ham, separable lean & 0.7 & 48.9 \\
\hline
\end{tabular}

${ }^{1}$ Food codes and descriptions correspond to those of the Standard Tables of Food Composition, 4th revised edition in Japan by Science and Technology Agency.

${ }^{2}$ Data on subjects in Ishikawa PHC (14-day data) were counted twice for (28-day data). 
Table 11. Cumulative $\%$ contribution of the top 20 foods for fat assessed by DR

\begin{tabular}{|c|c|c|c|}
\hline Food code ${ }^{1}$ & Description & g/day & $\begin{array}{c}\text { Cumulative }^{2} \\
\text { percent }\end{array}$ \\
\hline \multicolumn{4}{|c|}{ Men $(n=102)$} \\
\hline $5-15$ & Fats and oils/Vegetable oils/Vegetable oil, mixed & 10.5 & 17.8 \\
\hline $10-5 a$ & Chicken egg/Whole egg/fresh & 4.3 & 25.0 \\
\hline $11-2$ & Liquid milk/Ordinary liquid milk & 3.3 & 30.6 \\
\hline $1-41 d$ & Rice (Paddy rice)/Grains/Well-milled rice & 3.0 & 35.7 \\
\hline $9-70 \mathrm{a}$ & Pork and separable fat/Belly & 2.0 & 39.1 \\
\hline $7-21 a$ & Tofu and Abura-age/Tofu, soybean curd & 1.7 & 41.9 \\
\hline $17-10 \mathrm{a}$ & Seasonings/Mayonnaise/Whole egg type & 1.5 & 44.5 \\
\hline $7-32 c$ & Miso/Rice-koji Miso/Dark yellow type & 1.1 & 46.3 \\
\hline $8-84 a$ & Mackerel/Raw/Uncooked & 1.0 & 48.0 \\
\hline $8-95 a$ & Pacific saury/Raw/Uncooked & 0.9 & 49.6 \\
\hline $9-13 b$ & Beef and separable fat/Frank, Plate & 0.9 & 51.1 \\
\hline $7-22$ & Tofu and Abura-age/Okinawa-tofu & 0.8 & 52.5 \\
\hline $9-49 b$ & Chicken and separable fat/Thigh/Broiler & 0.8 & 53.8 \\
\hline $9-47 b$ & Chicken and separable fat/Breast & 0.8 & 55.1 \\
\hline $7-25$ & Tofu and Abura-age/Abura-age & 0.7 & 56.3 \\
\hline $9-22$ & Beef and separable fat/Ground meat & 0.7 & 57.6 \\
\hline $9-68 \mathrm{a}$ & Pork and separable fat/Loin, separable lean & 0.7 & 58.8 \\
\hline $7-29$ & Natto, fermented soybeans/Itohiki-natto & 0.7 & 59.9 \\
\hline $11-28$ & Butter, salted & 0.7 & 61.0 \\
\hline $9-85 \mathrm{a}$ & Pork products/Bacon/Bacon & 0.6 & 62.0 \\
\hline \multicolumn{4}{|c|}{ Women $(n=113)$} \\
\hline $5-15$ & Fats and oils/Vegetable oils/Vegetable oil, mixed & 9.0 & 17.0 \\
\hline $11-2$ & Liquid milk/Ordinary liquid milk & 3.8 & 24.2 \\
\hline $10-5 a$ & Chicken egg/Whole egg/fresh & 3.6 & 31.0 \\
\hline $1-41 d$ & Rice (Paddy rice)/Grains/Well-milled rice & 1.9 & 34.6 \\
\hline $9-70 \mathrm{a}$ & Pork and separable fat/Belly & 1.9 & 38.1 \\
\hline $17-10 \mathrm{a}$ & Seasonings/Mayonnaise/Whole egg type & 1.7 & 41.3 \\
\hline $7-21 \mathrm{a}$ & Tofu and Abura-age/Tofu, soybean curd & 1.4 & 44.0 \\
\hline $7-32 \mathrm{c}$ & Miso/Rice-koji Miso/Dark yellow type & 0.9 & 45.7 \\
\hline $8-95 a$ & Pacific saury/Raw/Uncooked & 0.8 & 47.1 \\
\hline $8-84 a$ & Mackerel/Raw/Uncooked & 0.8 & 48.6 \\
\hline $9-49 b$ & Chicken and separable fat/Thigh/Broiler & 0.7 & 50.0 \\
\hline $7-25$ & Tofu and Abura-age/Abura-age & 0.7 & 51.3 \\
\hline $11-28$ & Butter, salted & 0.7 & 52.6 \\
\hline $7-22$ & Tofu and Abura-age/Okinawa-tofu & 0.7 & 53.9 \\
\hline $9-13 b$ & Beef and separable fat/Frank, Short plate & 0.6 & 55.0 \\
\hline $7-29$ & Natto, fermented soybeans/tohiki-natto & 0.6 & 56.2 \\
\hline $9-47 b$ & Chicken and separable fat/Breast & 0.6 & 57.3 \\
\hline 9-87f & Pork products/Sausage/Lyoner & 0.6 & 58.4 \\
\hline $9-85 a$ & Pork products/Bacon/Bacon & 0.6 & 59.5 \\
\hline $5-7 \mathrm{a}$ & Margarine/Soft type & 0.6 & 60.5 \\
\hline
\end{tabular}

' Food codes and descriptions correspond to those of the Standard Tables of Food Composition, 4th revised edition in Japan by Science and Technology Agency.

${ }^{2}$ Data on subjects in Ishikawa PHC (14-day data) were counted twice for (28-day data). 
Table 12. Cumulative $\%$ contribution of the top 20 foods for carbohydrate assessed by DR

\begin{tabular}{|c|c|c|c|}
\hline Food code ${ }^{1}$ & Description' & g/day & $\begin{array}{c}\text { Cumulative }^{2} \\
\text { percent }\end{array}$ \\
\hline \multicolumn{4}{|c|}{ Men $(n=102)$} \\
\hline $1-41 d$ & Rice (Paddy rice)/Grains/Well-milled rice & 173.8 & 54.9 \\
\hline $16-2 a$ & Beer/Pale & 6.6 & 56.9 \\
\hline $3-4 a$ & Sugar/Soft sugar/White,superior & 5.7 & 58.7 \\
\hline $11-2$ & Liquid milk/Ordinary liquid milk & 4.7 & 60.2 \\
\hline $1-26 a$ & Chinese noodle i.e., Lao Mien/Raw, dry form & 4.7 & 61.7 \\
\hline $2-11 a$ & Potatoes/Tuber/Raw & 4.6 & 63.2 \\
\hline $1-10 \mathrm{a}$ & Wheat flour/Soft flour/First grade & 4.4 & 64.6 \\
\hline $1-13 a$ & Wheat (Breads)/White bread/On the market & 4.4 & 65.9 \\
\hline $1-21 b$ & Japanese noodles/Udon/Boiled & 4.3 & 67.3 \\
\hline $7-32 \mathrm{c}$ & Miso/Rice-koji Miso/Dark yellow type & 4.1 & 68.6 \\
\hline $13-88$ & Apples/Raw fruit & 3.9 & 69.8 \\
\hline $16-1 b$ & Sake/First class & 3.0 & 70.7 \\
\hline $1-24 b$ & Japanese noodles/Somen Hiyamugi/Boiled & 2.9 & 71.7 \\
\hline $13-64$ & Bananas/Raw fruit & 2.2 & 72.3 \\
\hline $16-31$ & Other beverages/Coffee drink, canned & 2.1 & 73.0 \\
\hline $1-62 a$ & Dried buckwheat noodle/Raw, dry form & 2.0 & 73.6 \\
\hline $1-47$ & Mochi, glutinous rice cake & 1.8 & 74.2 \\
\hline $12-18 \mathrm{a}$ & Pumpkin and squash/Squash/Raw & 1.6 & 74.7 \\
\hline $4-24 \mathrm{e}$ & Japanese-style confectioneries/Manju & 1.6 & 75.2 \\
\hline $13-26 a$ & Japanese Persimmons/Raw fruit/Hard type & 1.6 & 75.7 \\
\hline \multicolumn{4}{|c|}{ Women $(n=113)$} \\
\hline $1-41 d$ & Rice (Paddy rice)/Grains/Well-milled rice & 111.5 & 43.4 \\
\hline $3-4 a$ & Sugar/Soft sugar/White, superior & 5.7 & 45.6 \\
\hline $11-2$ & Liquid milk/Ordinary liquid milk & 5.4 & 47.7 \\
\hline $1-13 a$ & Wheat (Breads)/White bread/On the market & 5.4 & 49.8 \\
\hline $13-88$ & Apples/Raw fruit & 4.8 & 51.7 \\
\hline $2-11 a$ & Potatoes/Tuber/Raw & 4.2 & 53.3 \\
\hline $1-10 a$ & Wheat flour/Soft flour/First grade & 4.1 & 54.9 \\
\hline $1-21 b$ & Japanese noodles/Udon/Boiled & 3.8 & 56.4 \\
\hline $1-26 \mathrm{a}$ & Chinese noodle i.e., Lao Mien/Raw, dry form & 3.7 & 57.8 \\
\hline $7-32 c$ & Miso/Rice-koji Miso/Dark yellow type & 3.5 & 59.2 \\
\hline $4-24 \mathrm{e}$ & Japanese-style confectioneries/Manju & 3.1 & 60.4 \\
\hline $13-64$ & Bananas/Raw fruit & 2.3 & 61.3 \\
\hline $1-24 b$ & Japanese noodles/Somen Hiyamugi/Boiled & 2.2 & 62.1 \\
\hline $12-18 a$ & Pumpkin and squash/Squash/Raw & 2.1 & 62.9 \\
\hline $2-5 \mathrm{a}$ & Sweet potatoes/Tuber/Raw & 2.0 & 63.7 \\
\hline $13-26 a$ & Japanese Persimmons/Raw fruit/Hard type & 2.0 & 64.5 \\
\hline $13-17 b$ & Satsuma mandarins/Raw fruit & 1.9 & 65.3 \\
\hline $4-46$ & Confectioneries/Shortcake & 1.8 & 66.0 \\
\hline $4-41 d$ & Confectioneries/Rice-cracker & 1.7 & 66.6 \\
\hline 4-54 & Japanese buns/An-pan, bean-jam bun & 1.7 & 67.3 \\
\hline
\end{tabular}

1 Food codes and descriptions correspond to those of the Standard Tables of Food Composition, 4th revised edition in Japan by Science and Technology Agency.

${ }^{2}$ Data on subjects in Ishikawa PHC (14-day data) were counted twice for (28-day data). 
Table 13. Cumulative $\%$ contribution of the top 20 foods for alcohol assessed by DR

\begin{tabular}{|c|c|c|c|}
\hline Food code ${ }^{1}$ & Description' & g/day & $\begin{array}{c}\text { Cumulative }^{2} \\
\text { percent }\end{array}$ \\
\hline \multicolumn{4}{|c|}{ Men $(n=102)$} \\
\hline $16-2 \mathrm{a}$ & Beer/Pale & 7.7 & 34.0 \\
\hline $16-1 b$ & Sake/First class & 7.5 & 67.4 \\
\hline $16-4 b$ & Shochu/25\% alcohol & 5.3 & 90.8 \\
\hline $16-5 \mathrm{a}$ & Whisky/Special class & 0.6 & 93.5 \\
\hline $16-5 b$ & Whisky/First class & 0.6 & 96.0 \\
\hline $16-1 c$ & Sake/Second class & 0.3 & 97.5 \\
\hline $16-17 \mathrm{a}$ & Mirin/Hon-mirin & 0.2 & 98.6 \\
\hline $16-4 c$ & Shochu/20\% alcohol & 0.1 & 99.0 \\
\hline $16-3 a$ & Wine/White & 0.1 & 99.2 \\
\hline $16-10$ & Umeshu & 0.0 & 99.4 \\
\hline $16-6 b$ & Brandy/First class & 0.0 & 99.6 \\
\hline $17-33 a$ & Sakekasu/Seisyu & 0.0 & 99.7 \\
\hline 16-. & Sake/Special class & 0.0 & 99.8 \\
\hline $16-3 b$ & Wine/Red & 0.0 & 99.9 \\
\hline $16-12$ & Sweet wine & 0.0 & 99.9 \\
\hline $16-4 a$ & Shochu/ $35 \%$ alcohol & 0.0 & 99.9 \\
\hline $16-18$ & Medical liqueur & 0.0 & 100.0 \\
\hline $16-16$ & Shiro-zake & 0.0 & 100.0 \\
\hline $16-6 c$ & Brandy/Second class & 0.0 & 100.0 \\
\hline--- & --- & & \\
\hline \multicolumn{4}{|c|}{ Women $(n=113)$} \\
\hline $16-2 \mathrm{a}$ & Beer/Pale & 0.5 & 33.5 \\
\hline $16-1 b$ & Sake/First class & 0.4 & 56.3 \\
\hline $16-1 \mathrm{c}$ & Sake/Second class & 0.3 & 72.8 \\
\hline $16-17 \mathrm{a}$ & Mirin/Hon-mirin & 0.2 & 85.8 \\
\hline $16-10$ & Umeshu & 0.0 & 88.4 \\
\hline $17-33 a$ & Sakekasu/Seisyu & 0.0 & 90.3 \\
\hline $16-3 a$ & Wine/White & 0.0 & 92.1 \\
\hline $16-4 c$ & Shochu/20\% alcohol & 0.0 & 94.0 \\
\hline $16-5 a$ & Whisky/Special class & 0.0 & 95.2 \\
\hline $16-18$ & Medical liqueur & 0.0 & 96.4 \\
\hline $16-6 a$ & Brandy/Special class & 0.0 & 97.3 \\
\hline $16-4 b$ & Shochu/25\% alcohol & 0.0 & 98.2 \\
\hline $16-4 a$ & Shochu/35\% alcohol & 0.0 & 98.8 \\
\hline $16-3 b$ & Wine/Red & 0.0 & 99.4 \\
\hline $16-12$ & Sweet wine & 0.0 & 99.9 \\
\hline $16-6 b$ & Brandy/First class & 0.0 & 99.9 \\
\hline $16-9$ & Rum & 0.0 & 100.0 \\
\hline $16-14 a$ & Curacao/Orange & 0.0 & 100.0 \\
\hline $16-6 c$ & Brandy/Second class & 0.0 & 100.0 \\
\hline--- & --- & & \\
\hline
\end{tabular}

' Food codes and descriptions correspond to those of the Standard Tables of Food Composition, 4th revised edition in Japan by Science and Technology Agency.

${ }^{2}$ Data on subjects in Ishikawa PHC (14-day data) were counted twice for (28-day data). 
Table 14. Cumulative \% contribution of the top 20 foods for calcium assessed by DR

\begin{tabular}{|c|c|c|c|}
\hline Food code' & Description' & $\mathrm{mg} /$ day & $\begin{array}{c}\text { Cumulative }^{2} \\
\text { percent }\end{array}$ \\
\hline \multicolumn{4}{|c|}{ Men $(n=102)$} \\
\hline $11-2$ & Liquid milk/Ordinary liquid milk & 104.2 & 16.7 \\
\hline $7-21 \mathrm{a}$ & Tofu and Abura-age/Tofu, soybean curd & 40.5 & 23.2 \\
\hline $7-32 c$ & Miso/Rice-koji Miso/Dark yellow type & 25.3 & 27.3 \\
\hline $10-5 \mathrm{a}$ & Chicken egg/Whole egg/fresh & 21.0 & 30.7 \\
\hline $8-31$ & Sardines/Boiled and dried & 15.2 & 33.1 \\
\hline $7-22$ & Tofu and Abura-age/Okinawa-tofu & 14.1 & 35.3 \\
\hline $1-41 d$ & Rice (Paddy rice)/Grains/Well-milled rice & 13.8 & 37.6 \\
\hline $12-24 a$ & Cabbage/Head/Raw & 11.6 & 39.4 \\
\hline $11-23$ & Cheeses/Process cheeses & 10.5 & 41.1 \\
\hline $12-56 a$ & Daikon, Japanese radish/Root/Raw & 9.7 & 42.7 \\
\hline $12-98 b$ & Nozawana/Leaves/Salted & 8.4 & 44.0 \\
\hline $12-94 a$ & Carrot/Root/Raw & 8.2 & 45.3 \\
\hline $12-32 \mathrm{a}$ & Komatsuna/Leaves/Raw & 8.1 & 46.6 \\
\hline $15-35 \mathrm{a}$ & Wakame/Dried/Dried & 7.9 & 47.9 \\
\hline $7-25$ & Tofu and Abura-age/Abura-age & 6.7 & 49.0 \\
\hline $7-27$ & Tofu and Abura-age/Kori-dofu & 6.4 & 50.0 \\
\hline $12-117 \mathrm{a}$ & Spinach/Leaves/Raw & 6.3 & 51.0 \\
\hline $7-29$ & Natto, fermented soybeans/Itohiki-natto & 6.2 & 52.0 \\
\hline $11-9 \mathrm{a}$ & Yogurt/Whole milk, unsweetened & 5.5 & 52.9 \\
\hline $16-31$ & Other beverages/Coffee drink, canned & 5.5 & 53.8 \\
\hline \multicolumn{4}{|c|}{ Women $(n=113)$} \\
\hline $11-2$ & Liquid milk/Ordinary liquid milk & 119.7 & 20.0 \\
\hline $7-21 \mathrm{a}$ & Tofu and Abura-age/Tofu, soybean curd & 33.2 & 25.5 \\
\hline $7-32 c$ & Miso/Rice-koji Miso/Dark yellow type & 21.6 & 29.1 \\
\hline $10-5 \mathrm{a}$ & Chicken egg/Whole egg/fresh & 17.5 & 32.0 \\
\hline $8-31$ & Sardines/Boiled and dried & 13.4 & 34.3 \\
\hline $7-22$ & Tofu and Abura-age/Okinawa-tofu & 11.0 & 36.1 \\
\hline $12-24 a$ & Cabbage/Head/Raw & 10.2 & 37.8 \\
\hline $11-23$ & Cheeses/Process cheeses & 9.8 & 39.4 \\
\hline $1-41 d$ & Rice (Paddy rice)/Grains/Well-milled rice & 8.8 & 40.9 \\
\hline $11-9 b$ & Yogurt/Partially skimmed, sweetened & 8.8 & 42.4 \\
\hline $12-56 a$ & Daikon, Japanese radish/Root/Raw & 8.6 & 43.8 \\
\hline $15-35 a$ & Wakame/Dried/Dried & 8.4 & 45.2 \\
\hline $12-32 \mathrm{a}$ & Komatsuna/Leaves/Raw & 8.1 & 46.6 \\
\hline $12-94 a$ & Carrot/Root/Raw & 7.6 & 47.8 \\
\hline $12-98 \mathrm{~b}$ & Nozawana/Leaves/Salted & 7.0 & 49.0 \\
\hline $7-25$ & Tofu and Abura-age/Abura-age & 6.5 & 50.1 \\
\hline $6-12 b$ & Nuts and Seeds/Sesame seeds/Roasted & 6.5 & 51.2 \\
\hline $12-117 \mathrm{a}$ & Spinach/Leaves/Raw & 6.4 & 52.2 \\
\hline $11-9 \mathrm{a}$ & Yogurt/Whole milk, unsweetened & 5.9 & 53.2 \\
\hline $7-29$ & Natto, fermented soybeans/Itohiki-natto & 5.6 & 54.2 \\
\hline
\end{tabular}

1 Food codes and descriptions correspond to those of the Standard Tables of Food Composition, 4th revised edition in Japan by Science and Technology Agency.

2 Data on subjects in Ishikawa PHC (14-day data) were counted twice for (28-day data). 
Table 15. Cumulative \% contribution of the top 20 foods for phosphorus assessed by DR

\begin{tabular}{|c|c|c|c|}
\hline Food code & Description' & $\mathrm{mg} /$ day & $\begin{array}{c}\text { Cumulative }^{2} \\
\text { percent }\end{array}$ \\
\hline \multicolumn{4}{|c|}{ Men $(n=102)$} \\
\hline $1-41 d$ & Rice (Paddy rice)/Grains/Well-milled rice & 321.1 & 22.7 \\
\hline $11-2$ & Liquid milk/Ordinary liquid milk & 93.8 & 29.3 \\
\hline $10-5 a$ & Chicken egg/Whole egg/fresh & 76.3 & 34.7 \\
\hline $7-32 c$ & Miso/Rice-koji Miso/Dark yellow type & 39.0 & 37.5 \\
\hline $8-150 a$ & Tuna/Bluefin tuna, raw/Lean meat & 32.5 & 39.8 \\
\hline $16-2 a$ & Beer/Pale & 29.9 & 41.9 \\
\hline $7-21 \mathrm{a}$ & Tofu and Abura-age/Tofu, soybean curd & 28.7 & 43.9 \\
\hline $17-3 a$ & Seasonings/Shoyu, soy-sauce/Koikuchi & 28.3 & 45.9 \\
\hline $8-206 a$ & Squid and cuttlefish/Raw/Uncooked & 19.4 & 47.3 \\
\hline $7-22$ & Tofu and Abura-age/Okinawa-tofu & 15.2 & 48.4 \\
\hline $2-11 \mathrm{a}$ & Potatoes/Tuber/Raw & 14.8 & 49.4 \\
\hline $8-78 a$ & Mild salted chum salmon/Uncooked & 14.0 & 50.4 \\
\hline $7-29$ & Natto, fermented soybeans/Itohiki-natto & 13.1 & 51.3 \\
\hline $11-23$ & Cheeses/Process cheeses & 12.2 & 52.2 \\
\hline $8-218$ & Spiny lobster, raw & 11.7 & 53.0 \\
\hline $9-47 b$ & Chicken and separable fat/Breast & 11.0 & 53.8 \\
\hline 8-31 & Sardines/Boiled and dried & 10.4 & 54.5 \\
\hline 8-77 & Salmon/Chum salmon, raw & 10.2 & 55.3 \\
\hline $8-84 a$ & Mackerel/Raw/Uncooked & 9.8 & 56.0 \\
\hline $8-95 a$ & Pacific saury/Raw/Uncooked & 9.1 & 56.6 \\
\hline \multicolumn{4}{|c|}{ Women $(n=113)$} \\
\hline $1-41 d$ & Rice (Paddy rice)/Grains/Well-milled rice & 205.9 & 17.6 \\
\hline $11-2$ & Liquid milk/Ordinary liquid milk & 107.7 & 26.8 \\
\hline $10-5 \mathrm{a}$ & Chicken egg/Whole egg/fresh & 63.8 & 32.2 \\
\hline $7-32 c$ & Miso/Rice-koji Miso/Dark yellow type & 33.3 & 35.0 \\
\hline $17-3 a$ & Seasonings/Shoyu, soy-sauce/Koikuchi & 24.6 & 37.1 \\
\hline $7-21 \mathrm{a}$ & Tofu and Abura-age/Tofu, soybean curd & 23.5 & 39.1 \\
\hline $8-150 \mathrm{a}$ & Tuna/Bluefin tuna, raw/Lean meat & 20.5 & 40.9 \\
\hline $8-206 a$ & Squid and cuttlefish/Raw/Uncooked & 13.8 & 42.1 \\
\hline $2-11 a$ & Potatoes/Tuber/Raw & 13.6 & 43.2 \\
\hline $8-78 a$ & Mild salted chum salmon/Uncooked & 12.7 & 44.3 \\
\hline $7-22$ & Tofu and Abura-age/Okinawa-tofu & 11.9 & 45.3 \\
\hline $7-29$ & Natto, fermented soybeans/Itohiki-natto & 11.9 & 46.3 \\
\hline $11-23$ & Cheeses/Process cheeses & 11.4 & 47.3 \\
\hline $8-31$ & Sardines/Boiled and dried & 9.2 & 48.1 \\
\hline $8-218$ & Spiny lobster, raw & 9.0 & 48.9 \\
\hline $8-77$ & Salmon/Chum salmon,raw & 8.9 & 49.6 \\
\hline $9-47 b$ & Chicken and separable fat/Breast & 8.6 & 50.4 \\
\hline $1-13 a$ & Wheat (Breads)/White bread/On the market & 7.8 & 51.0 \\
\hline $8-95 a$ & Pacific saury/Raw/Uncooked & 7.6 & 51.7 \\
\hline $11-9 b$ & Yogurt/Partially skimmed, sweetened & 7.5 & 52.3 \\
\hline
\end{tabular}

1 Food codes and descriptions correspond to those of the Standard Tables of Food Composition, 4th revised edition in Japan by Science and Technology Agency.

${ }^{2}$ Data on subjects in Ishikawa PHC (14-day data) were counted twice for (28-day data). 
Table 16. Cumulative $\%$ contribution of the top 20 foods for iron assessed by DR

\begin{tabular}{|c|c|c|c|}
\hline Food code' & Description' & $\mathrm{mg} /$ day & $\begin{array}{c}\text { Cumulative }^{2} \\
\text { percent }\end{array}$ \\
\hline \multicolumn{4}{|l|}{$\operatorname{Men}(n=102)$} \\
\hline $1-41 d$ & Rice (Paddy rice)/Grains/Well-milled rice & 1.1 & 8.9 \\
\hline $7-32 c$ & Miso/Rice-koji Miso/Dark yellow type & 0.8 & 15.4 \\
\hline $10-5 a$ & Chicken egg/Whole egg/fresh & 0.7 & 20.8 \\
\hline $7-21 \mathrm{a}$ & Tofu and Abura-age/Tofu, soybean curd & 0.5 & 24.5 \\
\hline $17-3 a$ & Seasonings/Shoyu,soy-sauce/Koikuchi & 0.5 & 28.1 \\
\hline $12-117 \mathrm{a}$ & Spinach/Leaves/Raw & 0.4 & 31.4 \\
\hline $8-150 \mathrm{a}$ & Tuna/Bluefin tuna, raw/Lean meat & 0.2 & 33.2 \\
\hline $7-29$ & Natto, fermented soybeans/Itohiki-natto & 0.2 & 34.9 \\
\hline $7-22$ & Tofu and Abura-age/Okinawa-tofu & 0.2 & 36.5 \\
\hline $12-94 a$ & Carrot/Root/Raw & 0.2 & 37.8 \\
\hline $9-80$ & Swine(Sweetbreads)/Liver & 0.2 & 39.1 \\
\hline $15-28$ & Hijiki/Boiled and dried & 0.2 & 40.4 \\
\hline $16-21 b$ & Green tea/Sencha, common grade/Infusion & 0.1 & 41.5 \\
\hline $2-11 a$ & Potatoes/Tuber/Raw & 0.1 & 42.6 \\
\hline $8-31$ & Sardines/Boiled and dried & 0.1 & 43.5 \\
\hline $12-24 a$ & Cabbage/Head/Raw & 0.1 & 44.4 \\
\hline $11-2$ & Liquid milk/Ordinary liquid milk & 0.1 & 45.2 \\
\hline $7-27$ & Tofu and Abura-age/Kori-dofu & 0.1 & 46.0 \\
\hline $12-56 \mathrm{a}$ & Daikon, Japanese radish/Root/Raw & 0.1 & 46.7 \\
\hline $7-25$ & Tofu and Abura-age/Abura-age & 0.1 & 47.5 \\
\hline \multicolumn{4}{|c|}{ Women $(n=113)$} \\
\hline $1-41 d$ & Rice (Paddy rice)/Grains/Well-milled rice & 0.7 & 6.5 \\
\hline $7-32 c$ & Miso/Rice-koji Miso/Dark yellow type & 0.7 & 12.9 \\
\hline $10-5 \mathrm{a}$ & Chicken egg/Whole egg/fresh & 0.6 & 18.0 \\
\hline $12-117 \mathrm{a}$ & Spinach/Leaves/Raw & 0.4 & 21.8 \\
\hline $17-3 a$ & Seasonings/Shoyu,soy-sauce/Koikuchi & 0.4 & 25.4 \\
\hline $7-21 \mathrm{a}$ & Tofu and Abura-age/Tofu, soybean curd & 0.4 & 28.8 \\
\hline $7-29$ & Natto, fermented soybeans/Itohiki-natto & 0.2 & 30.7 \\
\hline 7.22 & Tofu and Abura-age/Okinawa-tofu & 0.2 & 32.0 \\
\hline $12-94 a$ & Carrot/Root/Raw & 0.2 & 33.4 \\
\hline $15-28$ & Hijiki/Boiled and dried & 0.2 & 34.8 \\
\hline $16-21 b$ & Green tea/Sencha, common grade/Infusion & 0.1 & 36.1 \\
\hline $8-150 a$ & Tuna/Bluefin tuna, raw/Lean meat & 0.1 & 37.4 \\
\hline $2-11 \mathrm{a}$ & Potatoes/Tuber/Raw & 0.1 & 38.5 \\
\hline $11-2$ & Liquid milk/Ordinary liquid milk & 0.1 & 39.5 \\
\hline $1-13 a$ & Wheat (Breads)/White bread/On the market & 0.1 & 40.5 \\
\hline $8-31$ & Sardines/Boiled and dried & 0.1 & 41.5 \\
\hline $12-24 a$ & Cabbage/Head/Raw & 0.1 & 42.4 \\
\hline $7-25$ & Tofu and Abura-age/Abura-age & 0.1 & 43.2 \\
\hline $7-27$ & Tofu and Abura-age/Kori-dofu & 0.1 & 43.9 \\
\hline $9-80$ & Swine(Sweetbreads)/Liver & 0.1 & 44.7 \\
\hline
\end{tabular}

${ }^{1}$ Food codes and descriptions correspond to those of the Standard Tables of Food Composition, 4th revised edition in Japan by Science and Technology Agency.

2 Data on subjects in Ishikawa PHC (14-day data) were counted twice for (28-day data). 
Table 17. Cumulative $\%$ contribution of the top 20 foods for sodium assessed by DR

\begin{tabular}{|c|c|c|c|}
\hline Food code 1 & Description' & $\mathrm{mg} / \mathrm{day}$ & $\begin{array}{c}\text { Cumulative }^{2} \\
\text { percent }\end{array}$ \\
\hline \multicolumn{4}{|c|}{ Men $(n=102)$} \\
\hline $17-3 a$ & Seasonings/Shoyu, soy-sauce/Koikuchi & 1195 & 22.4 \\
\hline $7-32 c$ & Miso/Rice-koji Miso/Dark yellow type & 994 & 41.0 \\
\hline $17-2 a$ & Seasonings/Salts/Kitchen salt & 542 & 51.2 \\
\hline $17-8$ & Seasonings/Flavor seasoning & 171 & 54.4 \\
\hline $12-58$ & Daikon, Japanese radish/Takuan-zuke & 146 & 57.1 \\
\hline $8-78 \mathrm{a}$ & Mild salted chum salmon/Uncooked & 140 & 59.8 \\
\hline $13-14$ & Ume/Umeboshi, salted and dried & 100 & 61.6 \\
\hline $13-13$ & Ume/Umezuke, salted & 78 & 63.1 \\
\hline $17-11$ & Seasonings/Mentsuyu & 78 & 64.6 \\
\hline $12-25 b$ & Cucumber/Fruit/Salted & 75 & 66.0 \\
\hline $1-31 b$ & Precooked Chinese noodles/Dried by frying & 62 & 67.1 \\
\hline $17-16 b$ & Spices/Curry/Roux & 54 & 68.2 \\
\hline $8-81$ & Salmon/Salted roe & 54 & 69.2 \\
\hline $11-2$ & Liquid milk/Ordinary liquid milk & 52 & 70.1 \\
\hline $15-35 a$ & Wakame/Dried/Dried & 50 & 71.1 \\
\hline $10-5 \mathrm{a}$ & Chicken egg/Whole egg/fresh & 50 & 72.0 \\
\hline $1-13 a$ & Wheat (Breads)/White bread/On the market & 47 & 72.9 \\
\hline $12-98 b$ & Nozawana/Leaves/Salted & 43 & 73.7 \\
\hline $8-250$ & Fish paste products/Yaki-chikuwa, broiled & 40 & 74.5 \\
\hline $8-212$ & Squid and cuttlefish/Salted preserves & 36 & 75.1 \\
\hline \multicolumn{4}{|c|}{ Women $(n=113)$} \\
\hline $17-3 a$ & Seasonings/Shoyu, soy-sauce/Koikuchi & 1038 & 22.3 \\
\hline $7-32 c$ & Miso/Rice-koji Miso/Dark yellow type & 848 & 40.5 \\
\hline $17-2 a$ & Seasonings/Salts/Kitchen salt & 478 & 50.8 \\
\hline $17-8$ & Seasonings/Flavor seasoning & 141 & 53.8 \\
\hline $8-78 \mathrm{a}$ & Mild salted chum salmon/Uncooked & 127 & 56.6 \\
\hline $12-58$ & Daikon, Japanese radish/Takuan-zuke & 123 & 59.2 \\
\hline $13-14$ & Ume/Umeboshi, salted and dried & 77 & 60.9 \\
\hline $17-11$ & Seasonings/Mentsuyu & 74 & 62.5 \\
\hline $12-25 b$ & Cucumber/Fruit/Salted & 73 & 64.0 \\
\hline $13-13$ & Ume/Umezuke, salted & 62 & 65.4 \\
\hline $11-2$ & Liquid milk/Ordinary liquid milk & 60 & 66.6 \\
\hline $1-13 a$ & Wheat (Breads)/White bread/On the market & 58 & 67.9 \\
\hline $15-35 a$ & Wakame/Dried/Dried & 53 & 69.0 \\
\hline $17-16 b$ & Spices/Curry/Roux & 49 & 70.1 \\
\hline $8-81$ & Salmon/Salted roe & 45 & 71.1 \\
\hline $10-5 \mathrm{a}$ & Chicken egg/Whole egg/fresh & 41 & 72.0 \\
\hline $1-31 b$ & Precooked Chinese noodles/Dried by frying & 40 & 72.8 \\
\hline $17-1$ & Seasonings/Consomme, dried & 39 & 73.7 \\
\hline $8-250$ & Fish paste products/Yaki-chikuwa, broiled & 39 & 74.5 \\
\hline $12-98 b$ & Nozawana/Leaves/Salted & 36 & 75.3 \\
\hline
\end{tabular}

' Food codes and descriptions correspond to those of the Standard Tables of Food Composition, 4th revised edition in Japan by Science and Technology Agency.

2 Data on subjects in Ishikawa PHC (14-day data) were counted twice for (28-day data). 
Table 18. Cumulative \% contribution of the top 20 foods for potassium assessed by DR

\begin{tabular}{|c|c|c|c|}
\hline Food code ${ }^{1}$ & Description' & $\mathrm{mg} /$ day & $\begin{array}{c}\text { Cumulative }^{2} \\
\text { percent }\end{array}$ \\
\hline \multicolumn{4}{|c|}{ Men $(n=102)$} \\
\hline $1-41 d$ & Rice (Paddy rice)/Grains/Well-milled rice & 252 & 7.8 \\
\hline $11-2$ & Liquid milk/Ordinary liquid milk & 156 & 12.7 \\
\hline $2-11 a$ & Potatoes/Tuber/Raw & 121 & 16.5 \\
\hline $7-32 c$ & Miso/Rice-koji Miso/Dark yellow type & 86 & 19.1 \\
\hline $12-117 \mathrm{a}$ & Spinach/Leaves/Raw & 85 & 21.8 \\
\hline $12-94 a$ & Carrot/Root/Raw & 84 & 24.4 \\
\hline $17-3 a$ & Seasonings/Shoyu, soy-sauce/Koikuchi & 81 & 26.9 \\
\hline $12-56 a$ & Daikon, Japanese radish/Root/Raw & 78 & 29.3 \\
\hline $16-2 a$ & Fermented alcoholic beverages/Beer/Pale & 75 & 31.7 \\
\hline $12-24 a$ & Cabbage/Head/Raw & 57 & 33.4 \\
\hline $8-150 a$ & Tunas/Bluefin tuna, raw/Lean meat & 49 & 34.9 \\
\hline $10-5 a$ & Chicken egg/Whole egg/fresh & 46 & 36.4 \\
\hline $15-35 a$ & Wakame/Dried/Dried & 45 & 37.8 \\
\hline $7-29$ & Natto, fermented soybeans/Itohiki-natto & 45 & 39.2 \\
\hline $12-85$ & Tomatoes/Fruit & 40 & 40.4 \\
\hline $13-64$ & Bananas/Raw fruit & 37 & 41.6 \\
\hline $15-17$ & Konbu, kelp/Rishiri-konbu, dried & 36 & 42.7 \\
\hline $8-206 a$ & Squid and cuttlefish/Raw/Uncooked & 33 & 43.7 \\
\hline $12-18 \mathrm{a}$ & Pumpkin and squash/Squash/Raw & 32 & 44.7 \\
\hline $12-25 a$ & Cucumber/Fruit/Raw & 32 & 45.7 \\
\hline \multicolumn{4}{|c|}{ Women $(n=113)$} \\
\hline $11-2$ & Liquid milk/Ordinary liquid milk & 179 & 6.1 \\
\hline $1-41 d$ & Rice (Paddy rice)/Grains/Well-milled rice & 162 & 11.6 \\
\hline $2-11 a$ & Potatoes/Tuber/Raw & 111 & 15.3 \\
\hline $12-117 \mathrm{a}$ & Spinach/Leaves/Raw & 86 & 18.3 \\
\hline $12-94 \mathrm{a}$ & Carrot/Root/Raw & 78 & 20.9 \\
\hline $7-32 c$ & Miso/Rice-koji Miso/Dark yellow type & 73 & 23.4 \\
\hline $17-3 a$ & Seasonings/Shoyu, soy-sauce/Koikuchi & 70 & 25.8 \\
\hline $12-56 a$ & Daikon, Japanese radish/Root/Raw & 69 & 28.1 \\
\hline $12-85$ & Tomatoes/Fruit & 55 & 30.0 \\
\hline $12-24 a$ & Cabbage/Head/Raw & 50 & 31.6 \\
\hline $15-35 a$ & Wakame/Dried/Dried & 48 & 33.3 \\
\hline $7-29$ & Natto, fermented soybeans/Itohiki-natto & 41 & 34.7 \\
\hline $12-18 \mathrm{a}$ & Pumpkin and squash/Squash/Raw & 41 & 36.1 \\
\hline $13-88$ & Apples/Raw fruit & 39 & 37.4 \\
\hline $13-64$ & Bananas/Raw fruit & 39 & 38.7 \\
\hline $10-5 a$ & Chicken egg/Whole egg/fresh & 38 & 40.0 \\
\hline $15-17$ & Konbu, kelp/Rishiri-konbu, dried & 33 & 41.1 \\
\hline $2-5 a$ & Sweet potatoes/Tuber/Raw & 32 & 42.2 \\
\hline $12-25 \mathrm{a}$ & Cucumber/Fruit/Raw & 32 & 43.3 \\
\hline $8-150 a$ & Tuna/Bluefin tuna, raw/Lean meat & 31 & 44.3 \\
\hline
\end{tabular}

1 Food codes and descriptions correspond to those of the Standard Tables of Food Composition, 4th revised edition in Japan by Science and Technology Agency.

${ }^{2}$ Data on subjects in Ishikawa PHC (14-day data) were counted twice for (28-day data). 
Table 19. Cumulative $\%$ contribution of the top 20 foods for retinol assessed by DR

\begin{tabular}{|c|c|c|c|}
\hline Food code' & Description ${ }^{1}$ & $\mu \mathrm{g} /$ day & $\begin{array}{c}\text { Cumulative }^{2} \\
\text { percent }\end{array}$ \\
\hline \multicolumn{4}{|c|}{ Men $(n=102)$} \\
\hline $9-80$ & Swine (Sweetbreads)/Liver & 168.6 & 38.4 \\
\hline $10-5 a$ & Chicken egg/Whole egg/fresh & 72.5 & 54.9 \\
\hline $9-55$ & Chicken (Sweetbreads)/Liver & 67.4 & 70.2 \\
\hline $11-2$ & Liquid milk/Ordinary liquid milk & 28.1 & 76.7 \\
\hline $8-69$ & Sablefish/Raw & 20.6 & 81.4 \\
\hline $8-41$ & Eel/Kabayaki & 17.5 & 85.3 \\
\hline $11-28$ & Butter, salted & 4.0 & 86.3 \\
\hline $11-23$ & Cheeses/Process cheeses & 4.0 & 87.2 \\
\hline $8-8 \mathrm{a}$ & Conger myriaster (Brevoort) & 3.5 & 88.0 \\
\hline $8-77$ & Salmon/Chum salmon, raw & 2.9 & 88.6 \\
\hline $9-47 b$ & Chicken and separable fat/Breast & 2.5 & 89.2 \\
\hline $8-16$ & Angler fish/Liver & 2.3 & 89.7 \\
\hline $9-49 b$ & Chicken and separable fat/Thigh/Broiler & 2.1 & 90.2 \\
\hline $8-81$ & Salmon/Salted roe & 2.1 & 90.7 \\
\hline $8-95 a$ & Pacific saury/Raw/Uncooked & 2.1 & 91.2 \\
\hline $8-84 a$ & Mackerel/Raw/Uncooked & 1.8 & 91.6 \\
\hline $4-46$ & Confectioneries/Shortcake & 1.8 & 92.0 \\
\hline $8-124$ & Herring/Raw & 1.6 & 92.3 \\
\hline $8-241 a$ & Toyama squid/Raw/Uncooked & 1.5 & 92.7 \\
\hline $9-54$ & Chicken (Sweetbreads)/Heart & 1.5 & 93.0 \\
\hline \multicolumn{4}{|c|}{ Women $(n=113)$} \\
\hline $9-55$ & Chicken (Sweetbreads)/Liver & 96.6 & 26.1 \\
\hline $9-80$ & Swine (Sweetbreads)/Liver & 88.0 & 50.0 \\
\hline $10-5 a$ & Chicken egg/Whole egg/fresh & 60.6 & 66.4 \\
\hline $11-2$ & Liquid milk/Ordinary liquid milk & 32.3 & 75.1 \\
\hline $8-69$ & Sablefish/Raw & 14.2 & 78.9 \\
\hline $8-41$ & Eel/Kabayaki & 12.3 & 82.3 \\
\hline $9-26$ & Cattle (Sweetbreads)/Liver & 5.7 & 83.8 \\
\hline $11-28$ & Butter, salted & 4.2 & 84.9 \\
\hline $11-23$ & Cheeses/Process cheeses & 3.7 & 85.9 \\
\hline $4-46$ & Confectioneries/Shortcake & 3.5 & 86.9 \\
\hline $8-8 \mathrm{a}$ & Conger myriaster (Brevoort) & 2.8 & 87.7 \\
\hline $8-77$ & Salmon/Chum salmon, raw & 2.5 & 88.4 \\
\hline $9-49 b$ & Chicken and separable fat/Thigh/Broiler & 2.0 & 88.9 \\
\hline $4-7$ & Japanese-style confectioneries/Kasutera & 1.9 & 89.4 \\
\hline $9-47 b$ & Chicken and separable fat/Breast & 1.9 & 89.9 \\
\hline $8-81$ & Salmon/Salted roe & 1.8 & 90.4 \\
\hline $8-95 a$ & Pacific saury/Raw/Uncooked & 1.7 & 90.9 \\
\hline $9-54$ & Chicken (Sweetbreads)/Heart & 1.5 & 91.3 \\
\hline $8-84 a$ & Mackerel/Raw/Uncooked & 1.4 & 91.7 \\
\hline $11-9 \mathrm{a}$ & Yogurt/Whole milk, unsweetened & 1.3 & 92.0 \\
\hline
\end{tabular}

' Food codes and descriptions correspond to those of the Standard Tables of Food Composition, 4th revised edition in Japan by Science and Technology Agency.

${ }^{2}$ Data on subjects in Ishikawa PHC (14-day data) were counted twice for (28-day data). 
Table 20. Cumulative $\%$ contribution of the top 20 foods for carotene assessed by DR

\begin{tabular}{|c|c|c|c|}
\hline Food code & Description' & $\mu \mathrm{g} /$ day & $\begin{array}{c}\text { Cumulative }^{2} \\
\text { percent }\end{array}$ \\
\hline \multicolumn{4}{|l|}{ Men $(n=102)$} \\
\hline $12-94 a$ & Carrot/Root/Raw & 1539 & 46.9 \\
\hline $12-117 \mathrm{a}$ & Spinach/Leaves/Raw & 355 & 57.7 \\
\hline $12-93 a$ & Chinese chive/Leaves/Raw & 112 & 61.2 \\
\hline $12-98 b$ & Nozawana/Leaves/Salted & 103 & 64.3 \\
\hline $12-32 \mathrm{a}$ & Komatsuna/Leaves/Raw & 93 & 67.1 \\
\hline $12-18 \mathrm{a}$ & Pumpkin and squash/Squash/Raw & 74 & 69.4 \\
\hline $12-85$ & Tomatoes/Fruit & 68 & 71.5 \\
\hline $15-4$ & Purple laver/Toasted & 60 & 73.3 \\
\hline $12-39 \mathrm{a}$ & Garland chrysanthemum/Leaves/Raw & 57 & 75.1 \\
\hline $12-55 \mathrm{a}$ & Daikon, Japanese radish/Leaves/Raw & 44 & 76.4 \\
\hline $12-19 a$ & Leaf mustard/stems and leaves/Raw & 39 & 77.6 \\
\hline $13-79$ & Mangos/Raw fruit & 37 & 78.7 \\
\hline $15-5$ & Purple laver/Seasoned and dried & 35 & 79.8 \\
\hline $12-77 \mathrm{a}$ & Basella/Leaves/Raw & 33 & 80.8 \\
\hline $12-74 a$ & Chingentsuai/Leaves/Raw & 27 & 81.6 \\
\hline $15-35 \mathrm{a}$ & Wakame/Dried/Dried & 27 & 82.5 \\
\hline $12-72$ & Lettuce/Head lettuce, butter head type & 26 & 83.2 \\
\hline $12-114 a$ & Broccoli/Head/Raw & 26 & 84.0 \\
\hline $13-45$ & Watermelon/Raw fruit & 24 & 84.8 \\
\hline $12-76$ & New Zealand spinach/stems and leaves & 23 & 85.5 \\
\hline \multicolumn{4}{|c|}{ Women $(n=113)$} \\
\hline $12-94 a$ & Carrot/Root/Raw & 1422 & 44.6 \\
\hline $12-117 \mathrm{a}$ & Spinach/Leaves/Raw & 361 & 55.9 \\
\hline $12-93 a$ & Chinese chive/Leaves/Raw & 103 & 59.2 \\
\hline $12-18 \mathrm{a}$ & Pumpkin and squash/Squash/Raw & 93 & 62.1 \\
\hline $12-85$ & Tomatoes/Fruit & 93 & 65.0 \\
\hline $12-32 a$ & Komatsuna/Leaves/Raw & 92 & 67.9 \\
\hline $12-98 b$ & Nozawana/Leaves/Salted & 87 & 70.6 \\
\hline $15-4$ & Purple laver/Toasted & 67 & 72.7 \\
\hline $12-39 a$ & Garland chrysanthemum/Leaves/Raw & 54 & 74.4 \\
\hline $13-79$ & Mangos/Raw fruit & 47 & 75.9 \\
\hline $12-77 \mathrm{a}$ & Basella/Leaves/Raw & 43 & 77.3 \\
\hline $12-55 a$ & Daikon, Japanese radish/Leaves/Raw & 43 & 78.6 \\
\hline $15-5$ & Purple laver/Seasoned and dried & 31 & 79.6 \\
\hline $13-45$ & Watermelon/Raw fruit & 29 & 80.5 \\
\hline $15-35 a$ & Wakame/Dried/Dried & 29 & 81.4 \\
\hline $12-114 a$ & Broccoli/Head/Raw & 29 & 82.3 \\
\hline $12-74 a$ & Chingentsuai/Leaves/Raw & 27 & 83.1 \\
\hline $12-19 a$ & Leaf mustard/stems and leaves/Raw & 25 & 83.9 \\
\hline $12-113 a$ & Chard, Swiss chard/Leaves/Raw & 24 & 84.7 \\
\hline $12-25 \mathrm{a}$ & Cucumber/Fruit/Raw & 23 & 85.4 \\
\hline
\end{tabular}

${ }^{1}$ Food codes and descriptions correspond to those of the Standard Tables of Food Composition, 4th revised edition in Japan by Science and Technology Agency.

2 Data on subjects in Ishikawa PHC (14-day data) were counted twice for (28-day data). 
Table 21. Cumulative \% contribution of the top 20 foods for thiamin assessed by DR

\begin{tabular}{|c|c|c|c|}
\hline Food code & Description' & $\mathrm{mg} /$ day & $\begin{array}{c}\text { Cumulative }^{2} \\
\text { percent }\end{array}$ \\
\hline \multicolumn{4}{|c|}{ Men $(n=102)$} \\
\hline $1-41 d$ & Rice (Paddy rice)/Grains/Well-milled rice & 0.28 & 20.9 \\
\hline $9-68 \mathrm{a}$ & Pork and separable fat/Loin,separable lean & 0.05 & 25.0 \\
\hline $9-72 a$ & Pork/Inside ham, separable lean & 0.05 & 28.9 \\
\hline $9-70 \mathrm{a}$ & Pork/Belly without separable fat & 0.05 & 32.4 \\
\hline $11-2$ & Liquid milk/Ordinary liquid milk & 0.03 & 34.8 \\
\hline $10-5 a$ & Chicken egg/Whole egg/fresh & 0.03 & 37.1 \\
\hline $2-11 \mathrm{a}$ & Potatoes/Tuber/Raw & 0.03 & 39.3 \\
\hline $1-41 f$ & Enriched rice with embryo & 0.02 & 41.2 \\
\hline $7-21 \mathrm{a}$ & Tofu and Abura-age/Tofu, soybean curd & 0.02 & 43.0 \\
\hline $9-66 a$ & Pork/Boston butt, separable lean & 0.02 & 44.7 \\
\hline $9-86 c$ & Pork products/Ham/Loin & 0.02 & 46.1 \\
\hline $9-75 a$ & Pork and separable fat/Fillet & 0.02 & 47.5 \\
\hline $9-76$ & Pork and separable fat/Ground meat & 0.02 & 48.6 \\
\hline $12-117 \mathrm{a}$ & Spinach/Leaves/Raw & 0.01 & 49.7 \\
\hline $12-94 a$ & Carrot/Root/Raw & 0.01 & 50.9 \\
\hline $9-84$ & Pork products/Roasted pork & 0.01 & 51.9 \\
\hline $12-24 a$ & Cabbage/Head/Raw & 0.01 & 52.9 \\
\hline $13-17 b$ & Satsuma mandarins & 0.01 & 53.8 \\
\hline $7-22$ & Tofu and Abura-age/Okinawa-tofu & 0.01 & 54.7 \\
\hline $8-150 \mathrm{a}$ & Tuna/Bluefin tuna, raw/Lean meat & 0.01 & 55.6 \\
\hline \multicolumn{4}{|c|}{ Women $(n=113)$} \\
\hline $1-41 d$ & Rice (Paddy rice)/Grains/Well-milled rice & 0.15 & 15.7 \\
\hline $9-70 \mathrm{a}$ & Pork/Belly without separable fat & 0.04 & 19.6 \\
\hline $9-68 \mathrm{a}$ & Pork and separable fat/Loin, separable lean & 0.04 & 23.3 \\
\hline $9-72 a$ & Pork/Inside ham, separable lean & 0.04 & 26.9 \\
\hline $11-2$ & Liquid milk/Ordinary liquid milk & 0.03 & 30.1 \\
\hline $2-11 a$ & Potatoes/Tuber/Raw & 0.02 & 32.5 \\
\hline $10-5 a$ & Chicken egg/Whole egg/fresh & 0.02 & 34.8 \\
\hline $9-66 a$ & Pork/Boston butt, separable lean & 0.02 & 36.6 \\
\hline $7-21 \mathrm{a}$ & Tofu and Abura-age/Tofu, soybean curd & 0.02 & 38.3 \\
\hline $1-41 \mathrm{f}$ & Enriched rice with embryo & 0.02 & 40.0 \\
\hline $13-17 b$ & Satsuma mandarins & 0.01 & 41.5 \\
\hline $9-86 c$ & Pork products/Ham/Loin & 0.01 & 43.0 \\
\hline $9-75 a$ & Pork and separable fat/Fillet & 0.01 & 44.4 \\
\hline $12-117 \mathrm{a}$ & Spinach/Leaves/Raw & 0.01 & 45.7 \\
\hline $9-76$ & Pork and separable fav/Ground meat & 0.01 & 46.9 \\
\hline $12-94 a$ & Carrot/Root/Raw & 0.01 & 48.2 \\
\hline $12-24 a$ & Cabbage/Head/Raw & 0.01 & 49.2 \\
\hline $12-85$ & Tomatoes/Fruit & 0.01 & 50.3 \\
\hline $12-18 \mathrm{a}$ & Pumpkin and squash/Squash/Raw & 0.01 & 51.3 \\
\hline $8-78 \mathrm{a}$ & Mild salted chum salmon/Uncooked & 0.01 & 52.1 \\
\hline
\end{tabular}

' Food codes and descriptions correspond to those of the Standard Tables of Food Composition, 4th revised edition in Japan by Science and Technology Agency.

2 Data on subjects in Ishikawa PHC (14-day data) were counted twice for (28-day data). 
Table 22. Cumulative $\%$ contribution of the top 20 foods for riboflavin assessed by DR

\begin{tabular}{|c|c|c|c|}
\hline Food code & Description' & $\mathrm{mg} /$ day & $\begin{array}{c}\text { Cumulative }^{2} \\
\text { percent }\end{array}$ \\
\hline \multicolumn{4}{|c|}{$\operatorname{Men}(n=102)$} \\
\hline $10-5 \mathrm{a}$ & Chicken egg/Whole egg/fresh & 0.18 & 11.8 \\
\hline $11-2$ & Liquid milk/Ordinary liquid milk & 0.16 & 21.8 \\
\hline $1-41 d$ & Rice (Paddy rice)/Grains/Well-milled rice & 0.07 & 26.3 \\
\hline $16-2 a$ & Beer/Pale & 0.06 & 30.4 \\
\hline $9-80$ & Swine (Sweetbreads)/Liver & 0.05 & 33.4 \\
\hline $16-21 b$ & Green tea/Sencha, common grade/Infusion & 0.04 & 36.2 \\
\hline $7-29$ & Natto, fermented soybeans/Itohiki-natto & 0.04 & 38.7 \\
\hline $17-3 a$ & Seasonings/Shoyu, soy-sauce/Koikuchi & 0.04 & 41.2 \\
\hline $8-84 a$ & Mackerel/Raw/Uncooked & 0.03 & 43.3 \\
\hline $12-117 a$ & Spinach/Leaves/Raw & 0.03 & 45.0 \\
\hline $7-32 \mathrm{c}$ & Miso/Rice-koji Miso/Dark yellow type & 0.02 & 46.2 \\
\hline $8-95 a$ & Pacific saury/Raw/Uncooked & 0.02 & 47.5 \\
\hline $14-7 a$ & Shiitake/Dried/Uncooked & 0.02 & 48.4 \\
\hline $12-24 a$ & Cabbage/Head/Raw & 0.01 & 49.3 \\
\hline $9-70 \mathrm{a}$ & Pork/Belly without separable fat & 0.01 & 50.1 \\
\hline $8-60 a$ & Flatfish/Raw/Uncooked & 0.01 & 50.9 \\
\hline $8-78 \mathrm{a}$ & Mild salted chum salmon/Uncooked & 0.01 & 51.7 \\
\hline $9-49 b$ & Chicken and separable fat/Thigh/Broiler & 0.01 & 52.4 \\
\hline 9-72a & Pork/Inside ham, separable lean & 0.01 & 53.1 \\
\hline $16-31$ & Other beverages/Coffee drink, canned & 0.01 & 53.8 \\
\hline \multicolumn{4}{|c|}{ Women $(n=113)$} \\
\hline $11-2$ & Liquid milk/Ordinary liquid milk & 0.18 & 13.0 \\
\hline $10-5 \mathrm{a}$ & Chicken egg/Whole egg/fresh & 0.15 & 24.1 \\
\hline $16-21 b$ & Green tea/Sencha, common grade/Infusion & 0.04 & 27.3 \\
\hline $1-41 d$ & Rice (Paddy rice)/Grains/Well-milled rice & 0.04 & 30.5 \\
\hline $7-29$ & Natto, fermented soybeans//tohiki-natto & 0.04 & 33.0 \\
\hline $17-3 a$ & Seasonings/Shoyu, soy-sauce/Koikuchi & 0.03 & 35.5 \\
\hline $12-117 \mathrm{a}$ & Spinach/Leaves/Raw & 0.03 & 37.4 \\
\hline $8-84 a$ & Mackerel/Raw/Uncooked & 0.02 & 39.2 \\
\hline $9-80$ & Swine (Sweetbreads)/Liver & 0.02 & 41.0 \\
\hline $7-32 c$ & Miso/Rice-koji Miso/Dark yellow type & 0.02 & 42.2 \\
\hline $8-95 a$ & Pacific saury/Raw/Uncooked & 0.02 & 43.3 \\
\hline $14-7 \mathrm{a}$ & Shiitake/Dried/Uncooked & 0.01 & 44.4 \\
\hline $11-9 b$ & Yogurt/Partially skimmed, sweetened & 0.01 & 45.3 \\
\hline $9-55$ & Chicken (Sweetbreads)/Liver & 0.01 & 46.2 \\
\hline $16-26 b$ & Fermented tea/Oolong tea/Infusion & 0.01 & 47.1 \\
\hline $12-24 \mathrm{a}$ & Cabbage/Head/Raw & 0.01 & 48.0 \\
\hline $9-70 \mathrm{a}$ & Pork/Belly without separable fat & 0.01 & 48.8 \\
\hline $9-49 b$ & Chicken and separable fat/Thigh/Broiler & 0.01 & 49.6 \\
\hline $8-78 \mathrm{a}$ & Mild salted chum salmon/Uncooked & 0.01 & 50.4 \\
\hline $12-114 a$ & Broccoli/Head/Raw & 0.01 & 51.2 \\
\hline
\end{tabular}

1 Food codes and descriptions correspond to those of the Standard Tables of Food Composition, 4th revised edition in Japan by Science and Technology Agency.

${ }^{2}$ Data on subjects in Ishikawa PHC (14-day data) were counted twice for (28-day data). 
Table 23. Cumulative $\%$ contribution of the top 20 foods for niacin assessed by DR

\begin{tabular}{|c|c|c|c|}
\hline Food code ${ }^{\prime}$ & Description' & $\mathrm{mg} /$ day & $\begin{array}{c}\text { Cumulative }^{2} \\
\text { percent }\end{array}$ \\
\hline \multicolumn{4}{|l|}{ Men $(n=102)$} \\
\hline $1-41 d$ & Rice (Paddy rice)/Grains/Well-milled rice & 3.2 & 14.7 \\
\hline $16-2 a$ & Beer/Pale & 1.3 & 20.5 \\
\hline $8-150 a$ & Tuna/Bluefin tuna, raw/Lean meat & 1.2 & 25.8 \\
\hline $8-84 a$ & Mackerel/Raw/Uncooked & 0.6 & 28.6 \\
\hline $9-47 b$ & Chicken and separable fat/Breast & 0.6 & 31.3 \\
\hline $8-50$ & Skipjack and frigate mackerel/Skipjack, raw & 0.6 & 33.8 \\
\hline $2-11 a$ & Potatoes/Tuber/Raw & 0.5 & 36.0 \\
\hline $8-77$ & Salmon/Chum salmon, raw & 0.4 & 37.9 \\
\hline $8-78 \mathrm{a}$ & Mild salted chum salmon/Uncooked & 0.4 & 39.6 \\
\hline $9-68 \mathrm{a}$ & Pork and separable fat/Loin, separable lean & 0.4 & 41.2 \\
\hline 9-70a & Pork/Belly without separable fat & 0.3 & 42.8 \\
\hline $9-72 a$ & Pork/Inside ham, separable lean & 0.3 & 44.4 \\
\hline $8-206 a$ & Squid and cuttlefish/Raw/Uncooked & 0.3 & 45.9 \\
\hline $8-54$ & Skipjack/Katsuo-bushi, dried strip & 0.3 & 47.3 \\
\hline $8-153 c$ & Tuna/Canned with/Oil, solids and liquid & 0.3 & 48.8 \\
\hline $8-95 a$ & Pacific saury/Raw/Uncooked & 0.3 & 50.1 \\
\hline $7-32 c$ & Miso/Rice-koji Miso/Dark yellow type & 0.3 & 51.5 \\
\hline $14-10 \mathrm{a}$ & Shimeji/Shirotamogitake, raw/Uncooked & 0.2 & 52.5 \\
\hline $17-3 a$ & Seasonings/Shoyu, soy-sauce/Koikuchi & 0.2 & 53.5 \\
\hline $9-84$ & Pork products/Roasted pork & 0.2 & 54.5 \\
\hline \multicolumn{4}{|c|}{ Women $(n=113)$} \\
\hline $1-41 d$ & Rice (Paddy rice)/Grains/Well-milled rice & 2.1 & 12.2 \\
\hline $8-150 \mathrm{a}$ & Tuna/Bluefin tuna, raw/Lean meat & 0.7 & 16.5 \\
\hline $9-47 b$ & Chicken and separable fat/Breast & 0.5 & 19.2 \\
\hline $8-84 a$ & Mackerel/Raw/Uncooked & 0.4 & 21.8 \\
\hline $2-11 \mathrm{a}$ & Potatoes/Tuber/Raw & 0.4 & 24.5 \\
\hline $8-50$ & Skipjack and frigate mackerel/Skipjack, raw & 0.4 & 26.7 \\
\hline $8-77$ & Salmon/Chum salmon, raw & 0.4 & 28.8 \\
\hline $8-78 \mathrm{a}$ & Mild salted chum salmon/Uncooked & 0.3 & 30.8 \\
\hline 9-70a & Pork/Belly without separable fat & 0.3 & 32.7 \\
\hline 9-72a & Pork/Inside ham, separable lean & 0.3 & 34.3 \\
\hline $9-68 \mathrm{a}$ & Pork and separable fat/Loin, separable lean & 0.3 & 35.9 \\
\hline $8-153 c$ & Tuna/Canned with/Oil, solids and liquid & 0.3 & 37.5 \\
\hline $8-54$ & Skipjack/Katsuo-bushi, dried strip & 0.3 & 39.1 \\
\hline $7-32 c$ & Miso/Rice-koji Miso/Dark yellow type & 0.2 & 40.6 \\
\hline $8-95 a$ & Pacific saury/Raw/Uncooked & 0.2 & 42.1 \\
\hline $16-30 c$ & Other beverages/Coffee/Instant & 0.2 & 43.5 \\
\hline $8-206 a$ & Squid and cuttlefish/Raw/Uncooked & 0.2 & 44.9 \\
\hline $14-10 a$ & Shimeji/Shirotamogitake, raw/Uncooked & 0.2 & 46.2 \\
\hline $17-3 a$ & Seasonings/Shoyu, soy-sauce/Koikuchi & 0.2 & 47.3 \\
\hline $9-49 b$ & Chicken and separable fat/Thigh/Broiler & 0.2 & 48.4 \\
\hline
\end{tabular}

Food codes and descriptions correspond to those of the Standard Tables of Food Composition, 4th revised edition in Japan by Science and Technology Agency.

${ }^{2}$ Data on subjects in Ishikawa PHC (14-day data) were counted twice for (28-day data). 
Table 24. Cumulative \% contribution of the top 20 foods for vitamin C assessed by DR

\begin{tabular}{|c|c|c|c|}
\hline Food code' & Description & $\mathrm{mg} /$ day & $\begin{array}{c}\text { Cumulative }^{2} \\
\text { percent }\end{array}$ \\
\hline \multicolumn{4}{|c|}{ Men $(n=102)$} \\
\hline $12-24 a$ & Cabbage/Head/Raw & 11.9 & 9.0 \\
\hline $12-117 \mathrm{a}$ & Spinach/Leaves/Raw & 7.4 & 14.6 \\
\hline $13-26 \mathrm{a}$ & Japanese Persimmons/Raw fruit/Hard type & 6.9 & 19.9 \\
\hline $2-11 a$ & Potatoes/Tuber/Raw & 6.2 & 24.6 \\
\hline $12-92 \mathrm{a}$ & Bitter gourd/Fruit/Raw & 6.2 & 29.2 \\
\hline $16-21 b$ & Green tea/Sencha, common grade/Infusion & 5.9 & 33.7 \\
\hline $12-114 a$ & Broccoli/Head/Raw & 5.8 & 38.1 \\
\hline $12-56 a$ & Daikon, Japanese radish/Root/Raw & 4.9 & 41.7 \\
\hline $13-17 b$ & Satsuma mandarins & 4.2 & 44.9 \\
\hline $12-108 \mathrm{a}$ & Sweet pepper/Fruit/Raw & 4.0 & 47.9 \\
\hline $12-85$ & Tomatoes/Fruit & 3.5 & 50.6 \\
\hline $12-18 \mathrm{a}$ & Pumpkin and squash/Squash/Raw & 3.4 & 53.2 \\
\hline $16-35$ & Other beverages/Powdered soft drinks & 3.4 & 55.7 \\
\hline $12-98 b$ & Nozawana/Leaves/Salted & 3.0 & 57.9 \\
\hline $12-101 \mathrm{a}$ & Chinese cabbage/Head/Raw & 2.6 & 59.9 \\
\hline $12-32 \mathrm{a}$ & Komatsuna/Leaves/Raw & 2.1 & 61.5 \\
\hline $13-33$ & Guavas/Raw fruit & 2.0 & 63.0 \\
\hline $12-25 a$ & Cucumber/Fruit/Raw & 2.0 & 64.5 \\
\hline $13-6$ & Strawberries/Raw fruit & 1.6 & 65.7 \\
\hline $9-86 c$ & Pork products/Ham/Loin & 1.5 & 66.9 \\
\hline \multicolumn{4}{|c|}{ Women $(n=113)$} \\
\hline $12-24 \mathrm{a}$ & Cabbage/Head/Raw & 10.5 & 7.5 \\
\hline $13-26 a$ & Japanese Persimmons/Raw fruit/Hard type & 8.8 & 13.8 \\
\hline $12-117 \mathrm{a}$ & Spinach/Leaves/Raw & 7.6 & 19.2 \\
\hline $12-114 a$ & Broccoli/Head/Raw & 6.4 & 23.8 \\
\hline $13-17 b$ & Satsuma mandarins & 5.9 & 28.0 \\
\hline $16-21 b$ & Green tea/Sencha, common grade/Infusion & 5.9 & 32.3 \\
\hline $2-11 \mathrm{a}$ & Potatoes/Tuber/Raw & 5.7 & 36.3 \\
\hline $13-33$ & Guavas/Raw fruit & 5.1 & 40.0 \\
\hline $12-85$ & Tomatoes/Fruit & 4.7 & 43.4 \\
\hline $12-92 a$ & Bitter gourd/Fruit/Raw & 4.5 & 46.6 \\
\hline $12-56 a$ & Daikon, Japanese radish/Root/Raw & 4.3 & 49.7 \\
\hline $12-18 \mathrm{a}$ & Pumpkin and squash/Squash/Raw & 4.3 & 52.7 \\
\hline $12-108 \mathrm{a}$ & Sweet pepper/Fruit/Raw & 3.6 & 55.3 \\
\hline $13-6$ & Strawberries/Raw fruit & 3.1 & 57.5 \\
\hline $16-35$ & Other beverages/Powdered soft drinks & 3.0 & 59.7 \\
\hline $12-98 b$ & Nozawana/Leaves/Salted & 2.5 & 61.4 \\
\hline 12-101a & Chinese cabbage/Head/Raw & 2.4 & 63.1 \\
\hline $12-32 \mathrm{a}$ & Komatsuna/Leaves/Raw & 2.1 & 64.6 \\
\hline $2-5 a$ & Sweet potatoes/Tuber/Raw & 2.1 & 66.1 \\
\hline $12-25 \mathrm{a}$ & Cucumber/Fruit/Raw & 2.0 & 67.5 \\
\hline
\end{tabular}

1 Food codes and descriptions correspond to those of the Standard Tables of Food Composition, 4th revised edition in Japan by Science and Technology Agency.

${ }^{2}$ Data on subjects in Ishikawa PHC (14-day data) were counted twice for (28-day data). 
Table 25. Cumulative \% contribution of the top 20 foods for cholesterol assessed by DR

\begin{tabular}{|c|c|c|c|}
\hline Food code ${ }^{1}$ & Description $^{1}$ & $\mathrm{mg} /$ day & $\begin{array}{c}\text { Cumulative }^{2} \\
\text { percent }\end{array}$ \\
\hline \multicolumn{4}{|l|}{$\operatorname{Men}(n=102)$} \\
\hline $10-5 \mathrm{a}$ & Chicken egg/Whole egg/fresh & 179.3 & 42.9 \\
\hline $8-206 a$ & Squid and cuttlefish/Raw/Uncooked & 34.2 & 51.1 \\
\hline $11-2$ & Liquid milk/Ordinary liquid milk & 11.5 & 53.9 \\
\hline $8-81$ & Salmon/Salted roe & 7.2 & 55.6 \\
\hline $8-218$ & Spiny lobster, raw & 6.8 & 57.2 \\
\hline $8-150 a$ & Tuna/Bluefin tuna, raw/Lean meat & 5.8 & 58.6 \\
\hline $9-49 b$ & Chicken and separable fat/Thigh/Broiler & 5.0 & 59.8 \\
\hline $9-47 b$ & Chicken and separable fat/Breast & 4.9 & 61.0 \\
\hline $8-118 a$ & Salted pollack roe/Uncooked & 4.5 & 62.1 \\
\hline $8-78 a$ & Mild salted chum salmon/Uncooked & 4.2 & 63.1 \\
\hline 9-70a & Pork/Belly without separable fat & 3.9 & 64.0 \\
\hline $10-14$ & Chicken egg/Tamago-dofu & 3.6 & 64.9 \\
\hline $8-95 a$ & Pacific saury/Raw/Uncooked & 3.4 & 65.7 \\
\hline $8-84 a$ & Mackerel/Raw/Uncooked & 3.4 & 66.5 \\
\hline $9-80$ & Swine (Sweetbreads)/Liver & 3.2 & 67.3 \\
\hline $8-77$ & Salmon/Chum salmon,raw & 3.2 & 68.1 \\
\hline $9-13 b$ & Beef and separable fat/Frank, Short plate & 3.1 & 68.8 \\
\hline $4-46$ & Western-style confectioneries/Shortcake & 3.1 & 69.5 \\
\hline $8-109$ & Sea breams/Crimson sea bream, raw & 3.0 & 70.3 \\
\hline $9-68 \mathrm{a}$ & Pork and separable fat/Loin, separable lean & 2.9 & 71.0 \\
\hline \multicolumn{4}{|c|}{ Women $(n=113)$} \\
\hline $10-5 \mathrm{a}$ & Chicken egg/Whole egg/fresh & 149.9 & 42.1 \\
\hline $8-206 a$ & Squid and cuttlefish/Raw/Uncooked & 24.3 & 48.9 \\
\hline $11-2$ & Liquid milk/Ordinary liquid milk & 13.2 & 52.6 \\
\hline $8-81$ & Salmon/Salted roe & 6.1 & 54.3 \\
\hline $4-46$ & Western-style confectioneries/Shortcake & 6.1 & 56.0 \\
\hline $8-218$ & Spiny lobster, raw & 5.2 & 57.5 \\
\hline $4-44$ & Foreign-style confectioneries/Cupcake & 4.8 & 58.8 \\
\hline $9-49 b$ & Chicken and separable fat/Thigh/Broiler & 4.8 & 60.2 \\
\hline $4-7$ & Japanese-style confectioneries/Kasutera & 4.6 & 61.5 \\
\hline $8-78 \mathrm{a}$ & Mild salted chum salmon/Uncooked & 3.9 & 62.5 \\
\hline $9-47 b$ & Chicken and separable fat/Breast & 3.8 & 63.6 \\
\hline $8-118 a$ & Salted pollack roe/Uncooked & 3.7 & 64.7 \\
\hline 9-70a & Pork/Belly without separable fat & 3.7 & 65.7 \\
\hline $8-150 a$ & Tuna/Bluefin tuna, raw/Lean meat & 3.7 & 66.7 \\
\hline $10-14$ & Chicken egg/Tamago-dofu & 3.3 & 67.7 \\
\hline $8-95 a$ & Pacific saury/Raw/Uncooked & 2.9 & 68.5 \\
\hline $8-77$ & Salmon/Chum salmon, raw & 2.8 & 69.2 \\
\hline $9-55$ & Chicken (Sweetbreads)/Liver & 2.6 & 70.0 \\
\hline $8-84 a$ & Mackerel/Raw/Uncooked & 2.5 & 70.7 \\
\hline $4-48 b$ & Confectioneries/Cake doughnut & 2.3 & 71.3 \\
\hline
\end{tabular}

' Food codes and descriptions correspond to those of the Standard Tables of Food Composition, 4th revised edition in Japan by Science and Technology Agency.

${ }^{2}$ Data on subjects in Ishikawa PHC (14-day data) were counted twice for (28-day data). 